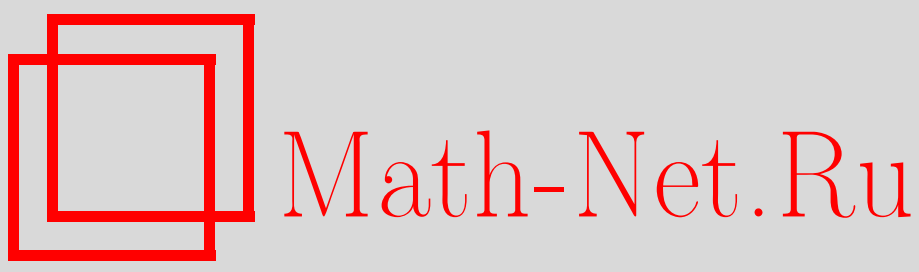

Б. А. Погорелов, М. А. Пудовкина, Орбитальные производные над кольцом вычетов. Часть І. Общие свойства, Матем. вопр. криптогр., 2014, том 5, выпуск 4, 99127

DOI: https://doi.org/10.4213/mvk137

Использование Общероссийского математического портала Math-Net.Ru подразумевает, что вы прочитали и согласны с пользовательским соглашением

http://www . mathnet.ru/rus/agreement

Параметры загрузки:

IP: 54.224 .135 .184

26 апреля 2023 г., 16:49:50

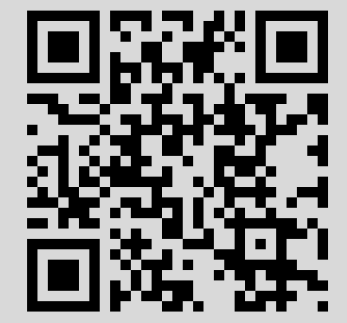


МАТЕМАТИЧЕСКИЕ ВОПРОСЫ КРИПТОГРАФИИ

2014 T. 5 № 4 C. 99-127

УДК 519.719.2

\title{
Орбитальные производные над кольцом вычетов. Часть I. Общие свойства
}

\author{
Б. А. Погорелов ${ }^{1}$, М. А. Пудовкина ${ }^{2}$ \\ ${ }^{1}$ Академия криптографии Российской Федерации, Москва \\ ${ }^{2}$ Национальный исследовательский ядерный университет (МИФИ), Москва
}

Получено 22.IV.2013

Для отображений $f: H \rightarrow F$, где $H$ и $F-$ множества, наделенные структурой конечных абелевых групп, предложено определение орбитальной производной $t$-го порядка, проистекающее из строения орбит подгрупп группы $H$. Описаны свойства орбитальной производной $t$-го порядка для преобразований над кольцом вычетов $\mathbb{Z}_{2^{n}}$.

Ключевые слова: орбитальная производная, абелевы группы, орбиты групп, невозможные наборы.

Orbital derivatives on residue rings. Part I. General properties B. A. Pogorelov ${ }^{1}$, M. A. Pudovkina ${ }^{2}$

${ }^{1}$ Academy of Cryptography of the Russian Federation, Moscow

${ }^{2}$ National Nuclear Research University, Moscow

Abstract. For mappings $f: H \rightarrow F$, where $H$ and $F$ are Abelian groups, a definition of the $t^{t h}$-order orbital derivative is introduced. The definition is based on structures of orbits of subgroups of $H$. Properties of the $t^{t h}$-order orbital derivative on the residue ring $\mathbb{Z}_{2^{n}}$ are described.

Keywords: orbital derivative, Abelian groups, orbits of groups, impossible sets.

Citation: Mathematical Aspects of Cryptography, 2014, vol. 5, no. 4, pp. 99-127 (Russian)

(c) 2014 Б. А. Погорелов, М. А. Пудовкина 


\section{1. Введение}

Функция зашифрования блочных шифрсистем при фиксированном ключе осуществляет биекцию множества $n$-мерных двоичных векторов в себя. При этом в слоях раундовых преобразований на части координат могут действовать преобразования, описываемые разными алгебраическими структурами. Слой наложения ключа чаще реализуется с помощью операции векторного сложения над полем $G F(2)$, а слой рассеивания - с помощью линейного преобразования векторного пространства $V_{n}=(G F(2))^{n}$. Поэтому в различных методах криптоанализа возникают производные двоичных функций и линеаризация над двоичными полями. Производная функции над полем $G F(2)$ по направлению $\delta \in V_{n}$ имеет меньшую алгебраическую степень, чем исходная функция (и в этом смысле является более "гладкой"), обладает большей симметрией (её группа инерции в группе сдвигов всегда содержит инволюцию сдвиг на вектор $\delta)$.

В случае элементарной абелевой группы (орбитальной группы векторного пространства $V_{n}$ ) операции сложения и вычитания совпадают. При этом производная двоичной функции в точке $x$ по направлению $\delta$ равна сумме её значений на орбите $\{x, x+\delta\}$ группы, порожденной сдвигом на вектор $\delta$. Аналогично может определяться и производная по направлению подпространства. В случае функций над другими алгебраическими структурами обычно рассматривается разность значений

$$
\Delta_{\delta} f(x)=f(x+\delta)-f(x)
$$

и в указанном смысле аналогия с двоичными функциями отсутствует.

Известно немало блочных шифрсистем, в которых слой наложения ключа или другие составляющие раундовой функции задаются с участием операции сложения в кольце вычетов $\mathbb{Z}_{2^{n}}$. Например, шифрсистемы ГOCT 28147-89, CAST-256, DFC, IDEA, LOKI97, MARS, RC2, RC5, RC6, SHA-ZAM. При их анализе в разностном методе рассматриваются разности элементов алфавитов открытого и шифрованного текстов в кольце вычетов. Так, в [1] (прежде всего в связи с разностным методом) для отображения $f: X \rightarrow X^{\prime}$ на паре абелевых групп $(X,+),\left(X^{\prime},+\right)$ производная по направлению $\delta \in X$ рассматривается как разность

$$
\Delta_{\delta} f(x)=\Delta_{\delta}^{(1)} f(x)=f(x+\delta)-f(x),
$$

a производная $r$-го порядка определяется индуктивно равенством $\Delta_{\delta_{r}, \ldots, \delta_{1}}^{(r)} f(x)=\Delta_{\delta_{r}}\left(\Delta_{\delta_{r-1}, \ldots, \delta_{1}}^{(r-1)} f(x)\right)$. В этом случае производная может уже не обладать большой группой инерции относительно действия пары 
групп, а для некоторых пар групп может не быть константой при сколь угодно больших $r$.

В криптографии нередко рассматриваются различные суммы элементов элементарных абелевых групп порядка $2^{n}$ (векторных пространств над полями характеристики 2): функция след, которая определяется как сумма элементов на орбитах группы, порожденной автоморфизмом Фробениуса; нулевые суммы и $k$-суммы [2], [3]; линейные структуры; разности и разности высшего порядка [1] в разностном методе и его обобщениях $[4]-[6]$.

Множество мощности $2^{n}$ можно наделять структурой различных абелевых групп. Эти группы могут иметь различные 2-ранги и существенно различаться по свойствам подгрупп и производных. Различаются они и строением групп автоморфизмов: в случае 2-ранга $n$ - это полная линейная группа $G L_{n}(2)$, а в случае 2-ранга 1 - это аддитивная группа кольца вычетов $\mathbb{Z}_{2^{n}}$. Для каждого $d \in\{2, \ldots, n-1\}$ существует абелева группа порядка $2^{n}$ и ранга $d$. Различны свойства таких групп и по структуре подгрупп. Так, в регулярной аддитивной группе кольца вычетов $\mathbb{Z}_{2^{n}}$ имеется единственная возрастающая система вложенных подгрупп порядков $1,2,2^{2}, \ldots, 2^{n}$ с такими же длинами орбит $1,2,2^{2}, \ldots, 2^{n}$. Единственная подгруппа порядка 2 в ней порождается сдвигом $\phi_{2^{n-1}}$ : $x \mapsto x+2^{n-1}\left(\bmod 2^{n}\right)$.

В настоящей работе для отображений $f: H \rightarrow F$, где $H$ и $F-$ множества, наделенные структурой конечных абелевых групп, предложено определение орбитальной производной $t$-го порядка, отличное от "классического" понятия конечной разности (см., например, [7]-[11]). Оно проистекает, прежде всего, из строения орбит подгрупп группы $H$, над которой рассматривается отображение $f: H \rightarrow F$. Значения орбитальных производных по аналогии с элементарными абелевыми 2-группами определяются суммированием значений отображения $f$ на орбитах группы $\left\langle 2^{n-t}\right\rangle$. В общем случае можно рассматривать не только сумму значений на орбитах подгрупп, но и значение произвольной симметрической функции на элементах орбиты. В настоящей работе рассматривается случай, когда $H$ и $F-$ кольца вычетов $\mathbb{Z}_{2^{n}}$ и $\mathbb{Z}_{2^{m}}$ соответственно, на элементах которых регулярно действуют соответствующие аддитивные группы.

Известно (см., например, [1]), что "классическая" производная $r$-го порядка отображения $g: V_{n} \rightarrow V_{m}, m \in\{1, \ldots, n\}$, принимает одно и то же значение для всех элементов из $V_{n}$ тогда и только тогда, когда алгебраическая степень каждой координатной функции отображения $g$ меньше $r$. Аналогом этого свойства является предложенное в работе понятие $\Delta_{+}-$ степени отображения $f: V_{n} \rightarrow V_{n}$ как такого наименьшего числа $r$, что 
орбитальная производная $r$-го порядка $\Delta_{+}^{(r)} f$ отображения $f$ принимает одно и то же значение на всех элементах $\mathbb{Z}_{2^{n}}$, при этом $r \leq n$. Описано множество всех отображений на $\mathbb{Z}_{2^{n}}$, у которых $\Delta_{+}$-степень равна $r \in\{1, \ldots, n\}$, и найдена его мощность. В качестве примера определена $\Delta_{+}$-степень степенного отображения.

Рассмотрен класс $(r, q)$-отображений, состоящий из всех таких отображений $g: \mathbb{Z}_{2^{n}} \rightarrow \mathbb{Z}_{2^{n}}$, что

$$
2^{r} q=\left|\left\{\alpha \in \mathbb{Z}_{2^{n}} \mid \Delta_{+}^{(r)} g(\alpha)=\beta\right\}\right|
$$

для всех $\beta \in\left\{\Delta_{+}^{(r)} g(\alpha) \mid \alpha \in \mathbb{Z}_{2^{n}}\right\}$. Класс $\left(r, 2^{n-r}\right)$-отображений состоит из всех отображений $g: \mathbb{Z}_{2^{n}} \rightarrow \mathbb{Z}_{2^{n}}$, у которых $\left|\left\{\Delta_{+} g(\alpha) \mid \alpha \in \mathbb{Z}_{2^{n}}\right\}\right|=1$, а их $\Delta_{+}$-степень не превосходит $r$. Единичная подстановка на $\mathbb{Z}_{2^{n}}$ имеет $\Delta_{+}$-степень, равную $n$, и является $(r, 1)$-отображением для каждого $r \in\{1, \ldots, n-1\}$. Отметим, что таким же свойством обладает и единичная подстановка на $V_{n}$ относительно последовательности "классических" производных по любой последовательности различных переменных.

Для произвольных $r, q$ описано множество всех $(r, q)$-отображений c указанием его мощности. Подобное описание множеств биективных $(r, q)$-отображений представляет собой более сложную задачу, которая нашла решение только для $(r, q) \in\left\{(1,1),\left(1,2^{n-1}\right),\left(2,2^{n-2}\right)\right\}$.

Исследуется структура множества значений, принимаемых орбитальной производной $r$-го порядка, для подстановок из $S\left(\mathbb{Z}_{2^{n}}\right)$. Описаны множества наборов значений (названные невозможными), которые производная $r$-го порядка не может принимать. Результаты могут представлять интерес в связи с линеаризацией сложных отображений над кольцом $\mathbb{Z}_{2^{n}}$.

\section{2. Обозначения и предварительные сведения}

Пусть $\mathbb{N}$ - множество всех натуральных чисел, $\mathbb{N}_{0}=\mathbb{N} \cup\{0\} ; \mathbb{Z}-$ кольцо целых чисел; $Z_{m}=\{0, \ldots, m-1\} \subset \mathbb{Z} ; \mathbb{Z}_{2^{n}}-$ кольцо вычетов по модулю $2^{n}$, элементы которого обозначаются числами $0, \ldots, 2^{n}-1$; при $d<n$ для $\alpha \in Z_{2^{n}}$ через $\alpha_{(d)}$ обозначаем такой элемент $\alpha_{(d)} \in Z_{2^{d}}$, что $\alpha_{(d)} \equiv a\left(\bmod 2^{d}\right)$, при этом $\alpha_{(0)}=0, x \mapsto x_{(d)}-$ соответствующее отображение; $A_{(d)}=\left\{\alpha_{(d)} \mid \alpha \in A\right\}$ для $A \subset \mathbb{Z} ; V_{n}$ - пространство $n$-мерных векторов над полем $G F(2)$, т.е. $V_{n}=(G F(2))^{n} ; \oplus$ - операция покоординатного сложения векторов в пространстве $V_{n} ; S(X)-$ множество всех подстановок на множестве $X ; X^{\times}=X \backslash\{0\}$, если 0 
- нейтральный элемент относительно заданной на $X$ бинарной операции; $\left\langle\beta_{1}, \ldots, \beta_{t}\right\rangle-$ подгруппа группы $X$ с некоторой бинарной операцией, порождённая элементами $\beta_{1}, \ldots, \beta_{t} \in X ; \bigoplus \alpha-$ сумма всех векторов из множества $W \subseteq V_{n} ;\left(\alpha_{1}, \ldots, \alpha_{t}\right) \equiv_{2^{n}} \quad\left(\beta_{1}, \ldots, \beta_{t}\right)$ - покоординатное равенство в кольце $\mathbb{Z}_{2^{n}}, t \geq 1 ; P\left(\mathbb{Z}_{2^{n}}\right)$ - множество всех отображений $\mathbb{Z}_{2^{n}} \rightarrow \mathbb{Z}_{2^{n}} ; \alpha^{g}=\alpha g=g(\alpha)$ - образ элемента $\alpha \in X$ при действии на него подстановкой $g \in S(X) ; \alpha^{G}=\left\{\alpha^{g} \mid g \in G\right\}, G \leq S(X)$; $A * B=\{\alpha * \beta \mid(\alpha, \beta) \in A \times B\}$ для бинарной операции $*$ группы $X$ и $A, B \subset X ;\|\alpha\|_{\chi}-$ вес Хемминга вектора $\alpha \in V_{n}$, т.е. число ненулевых координат; $\|\alpha\|-$ логарифмическая норма [12] элемента $\alpha \in \mathbb{Z}_{2^{n}}$, т.е. $\alpha=2^{\|\alpha\|} \beta, 2 \nmid \beta$; группы $\mathbb{Z}_{2^{n}}^{+}$и $V_{n}^{+}$- аддитивные группы кольца $\mathbb{Z}_{2^{n}}$ и векторного пространства $V_{n} ; a(x) \cdot b(x) \equiv_{2^{n}} c(x)$ есть сокращенная запись условия $a(x) \cdot b(x) \equiv c(x)\left(\bmod 2^{n}\right)$ для всех $x \in \mathbb{Z}_{2^{n}}$, где $a, b, c \in P\left(\mathbb{Z}_{2^{n}}\right)$, ". " - бинарная операция над числами в кольце $\mathbb{Z}$, представляющими соответствующие наименьшие неотрицательные вычеты по модулю $2^{n}$; запись $a *_{2^{n}} A$ означает, что элемент $a_{(n)}$ и множество $A_{(n)}$ находятся в отношении $* \in\{\in, \notin\}, a \in \mathbb{Z}, A \subset \mathbb{Z}$; запись $B *{ }_{2}{ }^{n} C$ означает, что множества $B_{(n)}$ и $C_{(n)}$ находятся в отношении $* \in\{\subseteq, \not \subset,=\}, B, C \subset \mathbb{Z} ; \mathbb{Z}_{2^{m}}^{c}$ - $c$-я декартова степень множества $\mathbb{Z}_{2^{m}}$;

$$
U_{n, t}(\delta)=\left\{\delta+2^{t} c \mid c \in Z_{2^{n-t}}\right\}=\delta+\left\langle 2^{t}\right\rangle\left(\bmod 2^{n}\right), \delta \in Z_{2^{n}} .
$$

Пусть $\Lambda=\left\{\left\{\lambda_{0}, \ldots, \lambda_{p-1}\right\}\right\}-$ мультимножество из $p$ элементов $\lambda_{0}, \ldots, \lambda_{p-1} \in Z_{2^{n}}$. Первичная спецификация (см., например, [10]) $\left[\theta_{0}^{t_{0}}, \ldots, \theta_{c-1}^{t_{c-1}}\right]$ мультимножества $\Lambda$ означает, что элемент $\theta_{i} \in Z_{2^{n}}$ встречается в мультимножестве $\Lambda$ равно $t_{i}$ раз, $t_{0}+\ldots+t_{c-1}=p$.

Вектор $\alpha=\left(\alpha_{n-1}, \ldots, \alpha_{1}, \alpha_{0}\right) \in V_{n}$ отождествляется с числом $\tilde{\alpha}=2^{n-1} \alpha_{n-1}+\ldots+2 \alpha_{1}+\alpha_{0}$. Тем самым на пространстве $V_{n}$ определяются операции сложения и умножения по модулю $2^{n}$, а над кольцом $\mathbb{Z}_{2^{n}}$ - операция $\oplus$ сложения векторов. Эти операции будем обозначать теми же символами, что и соответствующие операции над числами или векторами. Из контекста всегда будет ясно, о каких операциях идет речь.

Всюду ниже предполагается, что $n \geq 2$.

Для функции $f: V_{n} \rightarrow\{0,1\}$ производная по направлению $\beta_{1} \in V_{n}$ задаётся оператором $\Delta_{\oplus, \beta_{1}}$ :

$$
\Delta_{\oplus, \beta_{1}} f(x)=\Delta_{\oplus, \beta_{1}}^{(1)} f(x)=f\left(x \oplus \beta_{1}\right) \oplus f(x),
$$

a $r$-й (высшей) производной по направлениям $\beta_{1}, \ldots, \beta_{r}$ из $V_{n}$ называется сумма

$$
\Delta_{\oplus, \beta_{r}, \ldots, \beta_{1}}^{(r)} f(x)=\Delta_{\oplus, \beta_{r}}^{(1)} \ldots \Delta_{\oplus, \beta_{2}}^{(1)}\left(\Delta_{\oplus, \beta_{1}}^{(1)} f(x)\right) .
$$


Фактически, $r$-я производная задаётся суммированием на орбитах подгруппы группы сдвигов, порожденной сдвигами

$$
\phi_{\beta_{i}}: x \mapsto x \oplus \beta_{i}, \quad i=1,2, \ldots, r .
$$

Производные по направлению используются в методе высших разностей. Этот метод основан на том, что для векторов $\beta_{1}, \ldots, \beta_{r} \in V_{n}$ и функции $f: V_{n} \rightarrow\{0,1\}$ справедливы следующие свойства [1]:

1) $\Delta_{\oplus, \beta_{1}, \ldots, \beta_{r}}^{(r)} f(x)=\underset{\gamma \in\left\langle\beta_{1}, \ldots, \beta_{r}\right\rangle}{\bigoplus} f(x \oplus \gamma)$

2) $\Delta_{\oplus, \beta_{1}, \ldots, \beta_{r}}^{(r)} f(x)=0$, если векторы $\beta_{1}, \ldots \beta_{r}$ линейно зависимы;

3) $\operatorname{deg}(f)-r \geq \operatorname{deg}\left(\Delta_{\oplus, \beta_{1}, \ldots, \beta_{r}}^{(r)} f\right)$, если $\operatorname{deg}(f) \geq r$;

4) если $\Delta_{\oplus, \beta_{1}, \ldots, \beta_{r}}^{(r)} f$ не является константным отображением, то $\operatorname{deg}(f) \geq r$.

Центральная идея метода высших разностей состоит в применении двух подходов: разностного и линейного. В ряде случаев разностный метод сводится к нахождению инвариантов, которые сохраняются каким-то слоем раундовой функции, плохо рассеиваются другими слоями и слабо зависят от раундового ключа.

В методе высших разностей таким инвариантом является $r$-я производная по направлениям $\beta_{1}, \ldots, \beta_{r}$. Так, в методе высших разностей производные от функции зашифрования по направлению берутся до тех пор, пока получившееся преобразование не станет аффинным. После этого для нахождения битов раундового ключа применяется разновидность линейного метода. Метод успешно применён, например, в [5], [6]. На основе $r$-х производных в [13] предложено обобщение понятия бент-функции.

Рассмотрение сумм элементов позволяет надеяться, что они в меньшей степени зависят от частных свойств преобразований. Так, в последние годы $t$-суммы и нулевые суммы применялись при анализе криптосистем Hamsi, Keccak, Luffa [2], [3]. Пусть $s: V_{n} \rightarrow V_{m}$. Будем говорить, что множество $A=\left\{\alpha_{1}, \ldots, \alpha_{t}\right\}$ задаёт:

1) t-сумму, если $\alpha_{1}^{s} \oplus \ldots \oplus \alpha_{t}^{s}=0$;

2) нулевую $t$-сумму (или просто нулевую сумму), если $\bigoplus_{\alpha \in A} \alpha=0$ и $\bigoplus_{\alpha \in A} \alpha^{s}=0$ 
Нулевые $t$-суммы возникли в работе [14] при обобщении парадокса дней рождения следующим образом. Для $t$ мультимножеств $B_{1}, \ldots, B_{t}$, элементы которых выбраны случайно, равновероятно и независимо из $V_{n}$ (схема выбора с возвращением), требуется найти такие элементы $\beta_{1} \in$ $B_{1}, \ldots, \beta_{t} \in B_{t}$, что $\beta_{1} \oplus \ldots \oplus \beta_{t}=0$.

В настоящей работе вводится следующее определение орбитальной производной.

Определение 1. Орбитальной производной $r$-го порядка отображения $f: \mathbb{Z}_{2^{n}} \rightarrow \mathbb{Z}_{2^{m}}, n \in \mathbb{N}, m, r \in\{1, \ldots, n\}$, называется оператор $\Delta_{+}^{(r)}$, задаваемый формулой

$$
\Delta_{+}^{(r)} f(x) \equiv{ }_{2^{m}} \sum_{\beta \in U_{n, n-r}(x)} f(\beta) .
$$

Данное определение использует строение орбит подгрупп аддитивной группы кольца вычетов $\mathbb{Z}_{2^{n}}$. Значение орбитальной производной $\Delta_{+}^{(r)} f(x)$ равно сумме значений отображения $f$ на орбите циклической группы, порожденной сдвигом

$$
\phi_{2^{n-r}}: x \mapsto x+2^{n-r}\left(\bmod 2^{n}\right)
$$

и содержащей точку $x$. Сама орбита совпадает с множеством $U_{n, n-r}(x)$, являющимся смежным классом орбитальной группы кольца вычетов $\mathbb{Z}_{2^{n}}$ по подгруппе $\left\langle 2^{n-r}\right\rangle$.

Заметим, что для $n, r \in \mathbb{N}, m \in\{1, \ldots, n\}$ орбитальную производную отображения $f: \mathbb{Z}_{2^{n}} \rightarrow \mathbb{Z}_{2^{m}}$ по направлениям $\alpha_{1}, \ldots, \alpha_{r} \in \mathbb{Z}_{2^{n}}$ можно также определять равенством

$$
\Delta_{+, \alpha_{r}, \ldots, \alpha_{1}}^{\prime(r)} f(x)=\Delta_{+, \alpha_{r}}^{\prime(1)} \ldots \Delta_{+, \alpha_{2}}^{\prime(1)}\left(\Delta_{+, \alpha_{1}}^{\prime(1)} f(x)\right),
$$

где

$$
\Delta_{+, \alpha}^{\prime(1)} f(x) \equiv{ }_{2^{m}} \sum_{i=0}^{2^{n-d}-1} f(x+\alpha i),\|\alpha\|=d .
$$

Однако это определение легко сводится к определению 1.

В определении производных можно рассматривать не сумму значений функции от элементов, лежащих на орбите, а значение некоторой симметричной функции от элементов, лежащих на орбите. Аналогично можно ввести орбитальные производные для любой регулярной абелевой группы и ее подгрупп. В дальнейшем предполагается рассмотреть более общее понятие производной, связанное с суммированием на последовательностях возрастающих блоков импримитивности силовской подгруппы группы $S_{2^{n}}$, содержащей группы сдвигов кольца $\mathbb{Z}_{2^{n}}$ и пространства $V_{n}$. 


\section{3. Базовые свойства орбитальной производной $r$-го порядка}

Очевидно, что для любых $f \in P\left(\mathbb{Z}_{2^{n}}\right), \alpha \in \mathbb{Z}_{2^{n}}, r \in\{1, \ldots, n-1\}$ справедливо сравнение

$$
\Delta_{+}^{(r)} f(\alpha) \equiv_{2^{n}} \sum_{\gamma \in Z_{2^{r}}} f\left(\alpha_{(n-r)}+2^{n-r} \gamma\right) .
$$

Отсюда и из сравнения

$$
\Delta_{+}^{(r)} f\left(\alpha_{(n-r)}\right) \equiv_{2^{n}} \Delta_{+}^{(r)} f\left(\alpha_{(n-r)}+2^{n-r} \gamma\right),
$$

верного для всех $\gamma \in Z_{2^{r}}$, следует, что орбитальную производную $\Delta_{+}^{(r)} f$ можно рассматривать как отображение $\Delta_{+}^{(r)} f: \mathbb{Z}_{2^{n-r}} \rightarrow \mathbb{Z}_{2^{n}}$.

Если $f \in S\left(\mathbb{Z}_{2^{n}}\right)$, то

$$
\Delta_{+}^{(n)} f(x) \equiv_{2^{n}} \sum_{\beta \in \mathbb{Z}_{2^{n}}} f(\beta) \equiv_{2^{n}} \sum_{\beta \in Z_{2^{n-r}}} \Delta_{+}^{(r)} f(\beta) \equiv_{2^{n}} 0 .
$$

Справедливо также следующее рекуррентное соотношение, связывающее оператор $\Delta_{+}^{(r+d)}$ с оператором $\Delta_{+}^{(r)}$.

Лемма 1. Для $f \in P\left(\mathbb{Z}_{2^{n}}\right)$ выполняется сравнение

$$
\Delta_{+}^{(r+d)} f(x) \equiv_{2^{n}} \sum_{\beta \in Z_{2^{d}}} \Delta_{+}^{(r)} f\left(x+2^{n-r-d} \beta\right) .
$$

Доказательство. Действительно,

$$
\begin{aligned}
& \Delta_{+}^{(r+d)} f(x) \equiv_{2^{n}} \sum_{\beta \in Z_{2^{r+d}}} f\left(x+2^{n-r-d} \beta\right) \equiv_{2^{n}} \\
& \equiv_{2^{n}} \sum_{\beta_{1} \in Z_{2^{d}}} \sum_{\beta_{2} \in Z_{2} r} f\left(x+2^{n-r-d} \beta_{1}+2^{n-r} \beta_{2}\right) \equiv_{2^{n}} \\
& \equiv_{2^{n}} \sum_{\beta \in Z_{2^{d}}} \Delta_{+}^{(r)} f\left(x+2^{n-r-d} \beta\right) .
\end{aligned}
$$

Для биекции $f \in S\left(\mathbb{Z}_{2^{n}}\right)$ укажем соответствие между значениями орбитальной производной и некоторым разбиением множества $Z_{2^{n}}$ на подмножества по $2^{r}$ элементов. Значение $r$-й производной $\Delta_{+}^{(r)} f(x)$ в точке $\alpha \in \mathbb{Z}_{2^{n}}$ определяется орбитой $U_{n, n-r}(\alpha)$ группы $\left\langle 2^{n-r}\right\rangle$. Орбиты 
$U_{n, n-r}(0), \ldots, U_{n, n-r}(\alpha)$ задают разбиение множества $Z_{2^{n}}$ на блоки мощности $2^{r}$, а биекция $f$ индуцирует разбиение множества $Z_{2^{n}}$ на блоки $\left\{f(\beta) \mid \beta \in U_{n, n-r}(\alpha)\right\}$. В свою очередь, разбиению $\mathrm{B}_{n}^{(r)}=\left\{B_{0}, \ldots, B_{2^{n-r}-1}\right\}$ множества $Z_{2^{n}}$ на блоки $B_{0}, \ldots, B_{2^{n-r}-1}$ одинаковой мощности $2^{r}$ (с точностью до их упорядочивания) соответствует множество пар подстановок $(f, s) \in S\left(\mathbb{Z}_{2^{n}}\right) \times S\left(Z_{2^{n-r}}\right)$ со свойством $B_{\alpha^{s}}=\left\{f(\beta) \mid \beta \in U_{n, n-r}(\alpha)\right\}$ для каждого $\alpha \in Z_{2^{n-r}}$.

Положим $\beta_{i} \equiv_{2^{n}} \sum_{\alpha \in B_{i}} \alpha$ для $i=0, \ldots 2^{n-r}-1$. Разбиению $\mathrm{B}_{n}^{(r)}$ поставим в соответствие набор $\beta\left(\mathrm{B}_{n}^{(r)}\right)=\left(\beta_{0}, \ldots, \beta_{2^{n-r}-1}\right)$.

Описание множества всех подстановок, у которых мультимножество всех значений орбитальной производной $r$-го порядка имеет первичную спецификацию $\lambda$ (см., например, $[10])$, сводится к нахождению всех таких разбиений $\mathrm{B}_{n}^{(r)}$, что $\beta\left(\mathrm{B}_{n}^{(r)}\right)=\left(\gamma_{0}, \ldots, \gamma_{2^{n-r}-1}\right)$, где $\gamma_{0} \leq \gamma_{1} \leq \ldots \leq$ $\gamma_{2^{n-r}-1}$, и первичная спецификация мультимножества $\left\{\left\{\gamma_{0}, \ldots, \gamma_{2^{n-r}-1}\right\}\right\}$ есть $\lambda$.

Пусть $n \in \mathbb{N}, \quad r \in\{1, \ldots, n-1\}, X \subseteq P\left(\mathbb{Z}_{2^{n}}\right)$. Набор $\left(\theta_{0}, \ldots, \theta_{2^{n-r}-1}\right) \in Z_{2^{n}}^{2^{n-r}}$ назовём $X$-невозможным, если для каждого преобразования $g \in X$ не выполняется хотя бы одно сравнение

$$
\sum_{\delta \in U_{n, n-r}(i)} g(\delta) \equiv_{2^{n}} \theta_{i}, \quad i=0, \ldots, 2^{n-r}-1 .
$$

Набор называется $X$-возможным, если он не является $X$ невозможным. Невозможные наборы можно использовать на этапе отбраковки ложных ключей аналогично тому, как это осуществляется в методе невозможных разностей. Очевидно, что при $X=P\left(\mathbb{Z}_{2^{n}}\right)$ не существуют $P\left(\mathbb{Z}_{2^{n}}\right)$-невозможные наборы, а для $X=S\left(\mathbb{Z}_{2^{r}}\right)$ картина иная. В частности, естественное условие на набор $\left(\beta_{0}, \ldots, \beta_{2^{n-r}-1}\right) \in \mathbb{Z}_{2^{n}}^{2^{n-r}}$ следует из свойств подстановок из $S\left(\mathbb{Z}_{2^{n}}\right)$.

Лемма 2. При $n \in \mathbb{N}, r \in\{1, \ldots, n-1\}$ набор $\left(\beta_{0}, \ldots, \beta_{2^{n-r}-1}\right) \in \mathbb{Z}_{2^{n}}^{2^{n-r}}$ является $S\left(\mathbb{Z}_{2^{n}}\right)$-невозможниым, если

$$
\beta_{0}+\ldots+\beta_{2^{n-r}-1} \not \equiv_{2^{n}} 2^{n-1} .
$$

В частности, $\quad S\left(\mathbb{Z}_{2^{n}}\right)$-невозможным является набор $(\delta, \ldots, \delta) \in \mathbb{Z}_{2^{n}}^{2^{n-r}}, \quad \delta \not 2_{2^{r}} 2^{r-1}$.

Доказательство. Для любой подстановки $s \in S\left(\mathbb{Z}_{2^{n}}\right)$ выполняется 
сравнение

$$
\sum_{\alpha \in \mathbb{Z}_{2^{n}}} \alpha^{s} \equiv_{2^{n}} \sum_{i=1}^{2^{n}-1} i \equiv 2_{2^{n}} 2^{n-1} .
$$

Если набор $\left(\beta_{0}, \ldots, \beta_{2^{n-r}-1}\right)$ является $S\left(\mathbb{Z}_{2^{n}}\right)$-возможным, то величина $\beta_{0}+$ $\ldots+\beta_{2^{n-r}-1}\left(\bmod 2^{n}\right)$ есть сумма всех элементов $\mathbb{Z}_{2^{n}}$ и $\beta_{0}+\ldots+\beta_{2^{n-r}-1} \equiv_{2^{n}}$ $2^{n-1}$. Кроме того, если $S\left(\mathbb{Z}_{2^{n}}\right)$-невозможный набор $\left(\beta_{0}, \ldots, \beta_{2^{n-r}-1}\right) \in \mathbb{Z}_{2^{n}}^{2^{n-r}}$ таков, что $\beta_{0}=\ldots=\beta_{2^{n-r}-1}=\delta$, то из соотношения (1) следует, что $\delta 2^{n-r} \not \equiv_{2^{n}} 2^{n-1}$, т.е. $\delta \not \equiv_{2^{r}} 2^{r-1}$.

Из леммы 2 получаем, что для каждого $r \in\{1, \ldots, n-1\}$ набор $(\underbrace{0, \ldots, 0}_{2^{n-r}})$ является $S\left(\mathbb{Z}_{2^{n}}\right)$-невозможным.

\section{4. Степень отображений относительно $\Delta_{+}$}

Хорошо известно (см., например, [1]), что "классическая" производная $r$-го порядка отображения $g: V_{n} \rightarrow V_{m}, m \in\{1, \ldots, n\}$, принимает одно и то же значение для всех элементов пространства $V_{n}$ тогда, когда степень каждой координатной функции отображения $g$ меньше $r$. Аналогом этого является следующее понятие.

$\Delta_{+}$-степенъю $d_{+}(f)$ отображения $f: \mathbb{Z}_{2^{n}} \rightarrow \mathbb{Z}_{2^{m}}, m \leq n$, назовём такое наименьшее число $r \in \mathbb{N}$, что $\Delta_{+}^{(r)} f(x) \equiv_{2^{m}} \lambda$ при некотором $\lambda \in \mathbb{Z}_{2^{m}}$. Очевидно, что $d_{+}(f) \leq n$.

Вычислим $\Delta_{+}$-степень $d_{+}(f)$ многочлена $f(x)$ над $\mathbb{Z}_{2^{n}}$ и найдём условие, при котором $\Delta_{+}$-степень максимальна.

$$
\begin{gathered}
\text { Для многочлена } f(x) \equiv_{2^{n}} \sum_{i=0}^{d} a_{i} x^{i} \text { над } \mathbb{Z}_{2^{n}} \text { положим } \\
f^{\prime}(x) \equiv{ }_{2^{n}} \sum_{i=0}^{d-1}(i+1) a_{i+1} x^{i} .
\end{gathered}
$$

Утверждение 1. Пусть $n \geq 2, r \in\{1, \ldots, n-1\}, f \in P\left(\mathbb{Z}_{2^{n}}\right), d \in \mathbb{N} u$ $f(x) \equiv_{2^{n}} \sum_{i=0}^{d} a_{i} x^{i}$. Тогдa:

1) $\Delta_{+}^{(r)} f(x) \equiv{ }_{2^{n}} 2^{r} f(x)+2^{n-1} f^{\prime}(x)$; 
2) $d_{+}(f)=n$, если $\|a\|=0$, где $a \equiv 2_{2^{n}} a_{1}+\sum_{j=1}^{\lfloor d / 2\rfloor} a_{2 j}$.

Доказательство. 1. Далее понадобятся три леммы.

Лемма 3. Для $r \in\{1, \ldots, n-1\}, d \in \mathbb{N}$ справедливы соотношения

$$
2^{(n-r) d} \sum_{\beta \in Z_{2} r} \beta^{d} \equiv 2^{n} \begin{cases}0, & \text { если } r \geq 2, d \geq 2, \\ 2^{n-1}, & \text { если } d=1 \\ 2^{(n-1) d}, & \text { если } r=1\end{cases}
$$

Доказательство. Рассмотрим два случая: число $d$ чётно и число $d$ нечётно. Пусть $d$ чётно, $d \geq 2$. Тогда для любого $b \in \mathbb{N}$ справедливо соотношение

$$
\sum_{\beta \in Z_{2^{b}}} \beta^{d} \equiv_{2^{b}} \sum_{\beta \in Z_{2^{b-1}}} \beta^{d}+\sum_{\beta \in Z_{2^{b-1}}}\left(2^{b-1}+\beta\right)^{d} \equiv_{2^{b}} 2 \sum_{\beta \in Z_{2^{b-1}}} \beta^{d} .
$$

Из сравнения (2), применяя индукцию по числу $b$, имеем

$$
\sum_{\beta \in Z_{2^{b}}} \beta^{d} \equiv_{2^{b}} 2^{b-1}
$$

Заметим, что если $u_{1} \equiv_{2^{b}} u_{2}$, то $2^{c} u_{1} \equiv_{2^{b+c}} 2^{c} u_{2}$ для любого числа $c \in \mathbb{N}$. Тогда из соотношения (3) при $r=b$ получаем

$$
2^{(n-r) d} \sum_{\beta \in Z_{2^{r}}} \beta^{d} \equiv_{2^{n}} 2^{(n-r)+(n-r)(d-1)+r-1} \equiv_{2^{n}} 0,
$$

где $(n-r)(d-1) \geq 1$.

Пусть $d$ нечётно, $d \geq 1$. Тогда для любого $b \in \mathbb{N}$ справедливы сравнения

$$
\begin{aligned}
& \sum_{\beta \in Z_{2^{b}}} \beta^{d} \equiv_{2^{b}} 2^{(b-1) d}+\sum_{\beta \in Z_{2^{b-1}}} \beta^{d}+\sum_{\beta \in Z_{2^{b-1}}}\left(2^{b}-\beta\right)^{d} \equiv_{2^{b}} \\
& \equiv_{2^{b}} 2^{(b-1) d}+\sum_{\beta \in Z_{2^{b-1}}} \beta^{d}+\sum_{\beta \in Z_{2^{b-1}}}(-\beta)^{d} \equiv_{2^{b}} 2^{(b-1) d} \equiv_{2^{b}} \\
& \equiv_{2^{b}} \begin{cases}1, & \text { если } b=1, \\
2^{b-1}, & \text { если } d=1, \\
0, & \text { если } b \geq 2, d \geq 2 .\end{cases}
\end{aligned}
$$


В частности, при $b=r$ из соотношения (4) получаем, что

$$
2^{(n-r) d} \sum_{\beta \in Z_{2^{r}}} 2^{n} \beta^{d} \equiv_{2^{n}} \begin{cases}2^{(n-r) d+r} \equiv_{2^{n}} 0, & \text { если } r \geq 2, d \geq 2, \\ 2^{(n-r)+r-1} \equiv_{2^{n}} 2^{n-1}, & \text { если } d=1, \\ 2^{(n-1) d}, & \text { если } r=1 .\end{cases}
$$

Лемма 4. Пусть $n \geq 2, c \in \mathbb{N}, g \in P\left(\mathbb{Z}_{2^{n}}\right), g(x)=x^{c}$. Тогда

$$
\Delta_{+}^{(r)} g(x) \equiv_{2^{n}} \begin{cases}x^{c} 2^{r}+c x^{c-1} 2^{n-1}, & \text { если } r \in\{1, \ldots, n-1\} \\ 2^{n-1}, & \text { если } r=n, c \equiv_{2} 1 \\ 0, & \text { если } r=n, c \equiv_{2} 0 .\end{cases}
$$

Доказательство. Пусть $r \in\{1, \ldots, n-1\}$. Тогда

$$
\begin{gathered}
\Delta_{+}^{(r)} g(x) \equiv{ }_{2^{n}} \sum_{\beta \in Z_{2^{r}}}\left(x+2^{n-r} \beta\right)^{c} \equiv_{2^{n}} \sum_{\beta \in Z_{2^{r}}} \sum_{j=0}^{c}\left(\begin{array}{c}
c \\
j
\end{array}\right) x^{c-j}\left(2^{n-r} \beta\right)^{j} \equiv_{2^{n}} \\
\equiv_{2^{n}} \sum_{j=0}^{c}\left(\begin{array}{c}
c \\
j
\end{array}\right) x^{c-j} \cdot 2^{(n-r) j} \sum_{\beta \in Z_{2^{r}}} \beta^{j} .
\end{gathered}
$$

Из соотношения (5) и леммы 3 имеем

$$
\Delta_{+}^{(r)} g(x) \equiv 2_{2^{n}} \sum_{j=0}^{c}\left(\begin{array}{c}
c \\
j
\end{array}\right) x^{c-j} \cdot 2^{(n-r) j} \sum_{\beta \in Z_{2} r} \beta^{j} \equiv 2^{n} x^{c} 2^{r}+c x^{c-1} 2^{n-1} .
$$

Пусть $r=n$. Из доказательства леммы 3 следует, что для любого $b \in \mathbb{N}$ справедливо соотношение

$$
\sum_{\beta \in Z_{2^{b}}} \beta^{d} \equiv_{2^{b}} \begin{cases}2^{b-1}, & \text { если } d \equiv_{2} 0, \\ 0, & \text { если } d \equiv_{2} 1 .\end{cases}
$$

Значит, при $b=n$ имеем

$$
\Delta_{+}^{(n)} g(x) \equiv_{2^{n}} \sum_{\beta \in Z_{2^{n}}} \beta^{c} \equiv_{2^{n}} \begin{cases}2^{n-1}, & \text { если } c \equiv_{2} 0 \\ 0, & \text { если } c \equiv_{2} 1 .\end{cases}
$$


Лемма 5. Пусть $n \geq 2, c \in \mathbb{N}, r \in\{1, \ldots, n-1\}, g \in P\left(\mathbb{Z}_{2^{n}}\right), g(x) \equiv_{2^{n}}$ $a_{c} x^{c}+a_{0}, a_{c} \neq 0$. Тогда

$$
\Delta_{+}^{(r)} g(x) \equiv 2_{2^{n}} a_{c} x^{c} 2^{r}+a_{c} c x^{c-1} 2^{n-1}+2^{r} a_{0} .
$$

Доказательство. Доказательство следует из леммы 4 и того, что $\Delta_{+}^{(r)} a_{0}=2^{r} a_{0}$.

Таким образом, п.1 утверждения 1 следует из лемм 4 и 5.

2) Отметим, что

$$
\begin{gathered}
f^{\prime}(\beta) \equiv_{2} \begin{cases}\sum_{j=0}^{\lfloor(d-1) / 2\rfloor} a_{2 j+1}, & \text { если }\|\beta\|=0, \\
a_{1}, & \text { если }\|\beta\| \geq 1 .\end{cases} \\
f(\beta) \equiv_{2} \begin{cases}\sum_{j=0}^{d} a_{j}, & \text { если }\|\beta\|=0, \\
a_{0}, & \text { если }\|\beta\| \geq 1 .\end{cases}
\end{gathered}
$$

Из п.1 утверждения 1 имеем

$$
\Delta_{+}^{(n-1)} f(x) \equiv_{2^{n}} 2^{n-1}\left(f(x)+f^{\prime}(x)\right) .
$$

Так как

$$
f(\beta)+f^{\prime}(\beta) \equiv_{2} \begin{cases}a_{1}+a_{0}, & \text { если }\|\beta\| \geq 1, \\ \sum_{j=0}^{\lfloor d / 2\rfloor} a_{2 j}, & \text { если }\|\beta\|=0,\end{cases}
$$

то $\Delta_{+}^{(n-1)} f(0) \not 2_{2^{n}} \Delta_{+}^{(n-1)} f(1)$, если

$$
a_{1}+a_{0} \not \equiv_{2} \sum_{j=0}^{\lfloor d / 2\rfloor} a_{2 j},
$$

т.е.

$$
a_{1}+\sum_{j=1}^{\lfloor d / 2\rfloor} a_{2 j} \equiv_{2} 1 .
$$


Следствие 1. Для многочлена $f(x)$ над $\mathbb{Z}_{2^{n}}$ и любых $r_{1}, r_{2} \in\{1, \ldots, n\}$, $n \geq 2$, справедливо сравнение

$$
\Delta_{+}^{\left(r_{2}\right)}\left(\Delta_{+}^{\left(r_{1}\right)} f(x)\right) \equiv_{2^{n}} 2^{r_{1}+r_{2}} f(x) .
$$

Доказательство. Доказательство непосредственно следует из утверждения 1.

Таким образом, $\Delta_{+}$-степень $d_{+}(f)$ многочлена $f(x)$ над $\mathbb{Z}_{2^{n}}$ не зависит от его алгебраической степени $d$, если $d<n$. Кроме того, композиция операторов $\Delta_{+}^{\left(r_{1}\right)}, \Delta_{+}^{\left(r_{2}\right)}$ отображает многочлен над $\mathbb{Z}_{2^{n}}$ в многочлен, принимающий значения в идеале $\left\langle 2^{r_{1}+r_{2}}\right\rangle$ кольца $\mathbb{Z}_{2^{n}}$.

Пусть

$$
W_{j, n}=\left\{\alpha \in \mathbb{Z}_{2^{n}} \mid\|\alpha\|=j\right\}, j \in\{0, \ldots, n-1\} .
$$

Найдем число $\left|\left\{\Delta_{+}^{(r)} x^{c} \mid x \in \mathbb{Z}_{2^{n}}\right\}\right|$.

Утверждение 2. Пусть $n \geq 2, r \in\{1, \ldots, n-1\}, c \in\left\{1, \ldots, 2^{n-1}-1\right\}$, $\|c\|=b$, u $f(x)=x^{c} \in P\left(\mathbb{Z}_{2^{n}}\right)$. Тогда для $j \in\{0, \ldots, n-1\}$ орбитальная производная $\Delta_{+}^{(r)} f(x)$ на множестве $W_{j, n}$ принимает $2^{n-j-1} / m_{c, j, r}$ различных значений, причём каждое значение ровно $m_{c, j, r}$ раз, где

$$
m_{c, j, r}= \begin{cases}2^{r+j(c-1)}, & \text { если } b=0, r+j c<n, \\ 2^{n-j-1}, & \text { если } b=0, r+j c \geq n, \\ 2^{b+1+r+j(c-1)}, & \text { если } b \geq 1, j c \leq n-b-r-2, \\ 2^{n-j-1}, & \text { если } b \geq 1, n-b-r-1 \leq j c .\end{cases}
$$

Кроме того, на множестве $Z_{2^{n}}$ орбитальная производная $\Delta_{+}^{r} f$ принимает нулевое значение ровно $m_{r}$ раз, где $v=\lceil(n-r) / c\rceil$,

$$
m_{r}= \begin{cases}2^{n-v}, & \text { если } r \neq n-1, \\ 2^{n-1}, & \text { если } r=n-1, c \equiv_{2} 0, \\ 2^{n}, & \text { если } r=n-1, c \equiv_{2} 1 .\end{cases}
$$

Доказательство. Согласно лемме 5 ,

$$
\Delta_{+}^{(r)} f(x) \equiv_{2^{n}} 2^{r} x^{c}+2^{n-1} c x^{c-1} .
$$


Отметим, что

$$
\Delta_{+}^{(r)} f(x) \equiv 2_{2^{n}} \begin{cases}2^{r} x^{c}, & \text { если }\|c x\| \geqslant 1 \text { при } c>1, \\ 2^{r} x^{c}+2^{n-1}, & \text { если } c=1 \text { или }\|c x\|=0 \text { при } c>1 .\end{cases}
$$

Таким образом, $\Delta_{+}^{(r)} f(\alpha) \equiv_{2^{n}} \Delta_{+}^{(r)} f(\beta)$ для $\alpha, \beta \in \mathbb{Z}_{2^{n}}$ тогда и только тогда, когда

$$
2^{r} \alpha^{c} \equiv 2^{n} 2^{r} \beta^{c} .
$$

В этом случае, если $\alpha \in W_{p, n}$ для некоторого $p \in\{0, \ldots, n-1\}$, то $\beta \in W_{p, n}$. Тогда существуют такие нечётные числа $\beta, \delta \in Z_{2^{n-p}}^{\times}$, что $\alpha=2^{p} \beta, \gamma=2^{p} \delta$. Из сравнения (8) следует, что $\beta^{c} 2^{r+p c} \equiv_{2^{n}} \delta^{c} 2^{r+p c}$, т.е. $\left(\delta^{-1} \beta\right)^{c} 2^{r+p c} \equiv_{2^{n}} 2^{r+p c}$ и $\left(\delta^{-1} \cdot \beta\right) \in_{2^{n}} Z_{2^{n-p}}$.

Пусть $q \in \mathbb{N}, m_{c, q}^{(1)}-$ число решений в $\mathbb{Z}_{2^{q}}$ сравнения

$$
x^{c} \equiv_{2^{q}} 1 .
$$

Нетрудно убедиться (см., например, [15]), что все решения сравнения (9) принадлежат множеству $W_{0, n}$ и

$$
m_{c, q}= \begin{cases}1, & \text { если } b=0, \\ 2^{b+1}, & \text { если } q \geq 3, b \in\{1, \ldots, q-2\}, \\ 2^{q-1}, & \text { если } q \geq 2, b \in\{q-1, \ldots, n-1\} .\end{cases}
$$

Тогда из равенства (10) следует, что $m_{c, p, r}-$ число решений в множестве $\left\{1+2 t \mid t \in\left\{0, \ldots, 2^{n-p-1}-1\right\}\right\}$ сравнения $2^{r+p(c-1)} x^{c} \equiv_{2^{n-p}} 2^{r+p(c-1)}$. Таким образом, орбитальная производная $\Delta_{+}^{r} f$ на множестве $W_{p, n}$ принимает каждое значение ровно $m_{c, p, r}$ раз, а всего число различных значений равно $2^{n-p-1} / m_{c, p, r}$. Из соотношения (7) следует, что число решений уравнения $\Delta_{+}^{(r)} f(x) \equiv_{2^{n}} 0$ равно $m_{r}$.

Нетрудно убедиться, что орбитальная производная $r$-го порядка для экспоненциальной функции $a^{x}$ равна

$$
\Delta_{+}^{(r)} a^{x} \equiv 2^{n} 2^{r} a^{x}
$$

Множество всех подстановок из $S\left(\mathbb{Z}_{2^{n}}\right)$ с одним циклом длины $q \geq 2$ и $2^{n}-q$ единичными циклами обозначим $C_{q}^{(n, 1)}$.

Утверждение 3. Пусть $n \geq 2, r \in\{1, \ldots, n-2\} u q \leq 2^{n-r}-1$. Тогда $r<d_{+}(g)$ для любой подстановки $g \in C_{q}^{(n, 1)}$. 
Доказательство. Пусть $B=\left\{\beta_{0}, \ldots, \beta_{q-1}\right\} \subseteq \mathbb{Z}_{2^{n}}$,

$$
g: \beta_{i} \mapsto \beta_{i+1(\bmod q)}, i=0, \ldots, q-1 .
$$

Заметим, что если $\delta \in Z_{2^{n-r}}$ и $B \cap U_{n, n-r}(\delta)=\varnothing$, то

$$
\Delta_{+}^{(r)} g(\delta) \equiv_{2^{n}} 2^{r} \delta+2^{n-r} \sum_{j=0}^{2^{r}-1} j \equiv_{2^{n}} 2^{r} \delta+2^{n-1} .
$$

Положим $\lambda_{r}(\delta) \equiv 2_{2^{n}} 2^{r} \delta+2^{n-1}$. Ясно, что для различных элементов $\delta_{1}, \delta_{2} \in Z_{2^{n-r}}$. Таким образом, при $q \leq 2^{n-r}-2$ существуют два таких элемента $\theta_{1}, \theta_{2} \in Z_{2^{n-r}}$, что

$$
\begin{gathered}
B \cap U_{n, n-r}\left(\delta_{i}\right)=\varnothing, \\
\Delta_{+}^{r} g\left(\delta_{i}\right) \equiv_{2^{n}} \lambda_{r}\left(\theta_{i}\right), i=1,2 .
\end{gathered}
$$

Значит, $d_{+}(g)>r$.

Если $q=2^{n-r}-1$, то возможны два случая.

1) $B \cap U_{n, n-r}\left(\delta_{i}\right)=\varnothing$ для некоторых различных $\theta_{1}, \theta_{2} \in Z_{2^{n-r}}$. Тогда $d_{+}(g)>r$.

2 ) Существуют такие попарно различные $\delta_{0}, \ldots, \delta_{q}$ из $Z_{2^{n-r}}$, что $\beta_{j} \in$ $U_{n, n-r}\left(\delta_{j}\right)$ для всех $j \in\{0, \ldots, q-1\}, B \cap U_{n, n-r}\left(\delta_{q}\right)=\varnothing$ и

$$
\begin{gathered}
\Delta_{+}^{(r)} g\left(\delta_{j}\right) \equiv_{2^{n}} 2^{r} \delta_{j}+2^{n-1}+\beta_{j+1(\bmod q)}-\beta_{j}, j=0, \ldots, q-1, \\
\Delta_{+}^{(r)} g\left(\delta_{q}\right) \equiv_{2^{n}} 2^{r} \delta_{q}+2^{n-1} .
\end{gathered}
$$

Если $\Delta_{+}^{(r)} g\left(\delta_{0}\right) \equiv_{2^{n}} \ldots \equiv_{2^{n}} \Delta_{+}^{(r)} g\left(\delta_{q}\right)$, то

$$
\beta_{j+1(\bmod q)}-\beta_{j} \equiv_{2^{n}} 2^{r}\left(\delta_{q}-\delta_{j}\right), j=0, \ldots, q-1 .
$$

Очевидно, что

$$
\left|\left\{\delta_{q}-\delta_{j} \mid j=0, \ldots, q-1\right\}\right|==_{2^{n}} q .
$$

Поэтому

$$
\beta_{j+1(\bmod q)} \equiv_{2^{n}} 2^{r}\left(\delta_{q}-\delta_{j}\right)+\beta_{j}, j=0, \ldots, q-1 .
$$

Значит,

$$
\sum_{j=0}^{q-1} \beta_{j+1(\bmod q)} \equiv_{2^{n}} \sum_{j=0}^{q-1}\left(2^{r}\left(\delta_{q}-\delta_{j}\right)+\beta_{j}\right),
$$


T.e.

$$
\sum_{j=0}^{q-1} 2^{r}\left(\delta_{q}-\delta_{j}\right) \equiv_{2^{n}} 0 .
$$

Так как

$$
\begin{aligned}
& \sum_{j=0}^{q-1} 2^{r}\left(\delta_{q}-\delta_{j}\right) \equiv_{2^{n}} 2^{r} q \delta_{q}-2^{r} \sum_{j=0}^{q-1} \delta_{j} \equiv_{2^{n}} \\
& \equiv_{2^{n}}\left(2^{n-r}-1\right) 2^{r} \delta_{q}-2^{r-1} \cdot 2^{n-r}\left(2^{n-r}-1\right)+2^{r} \delta_{q} \equiv_{2^{n}} \\
& \equiv_{2^{n}}-2^{r} \delta_{q}+2^{n-1}+2^{r} \delta_{q} \equiv_{2^{n}} 2^{n-1},
\end{aligned}
$$

то получаем противоречие со сравнением (11).

Из утверждения 3 следует, что $d_{+}(f) \geq n-1$, если $f$ - транспозиция или цикл длины три.

\section{5. $(r, q)$-отображения}

Для $q \in \mathbb{N}$ отображение $f \in P\left(\mathbb{Z}_{2^{n}}\right)$ назовём $(r, q)$-отображснием, если

$$
2^{r} q=\left|\left\{\alpha \in \mathbb{Z}_{2^{n}} \mid \Delta_{+}^{(r)} f(\alpha)=\beta\right\}\right|
$$

для всех $\beta \in\left\{\Delta_{+}^{(r)} f(\alpha) \mid \alpha \in \mathbb{Z}_{2^{n}}\right\}$. Пусть $\mathrm{B}_{r, q}$ - множество всех $(r, q)$ отображений над $\mathbb{Z}_{2^{n}}$. Если для некоторого $q$ отображение $f$ является $(r, q)$ - отображением, то будем называть его также $r$-отображсением.

Для $f \in P\left(\mathbb{Z}_{2^{n}}\right)$ положим

$$
C_{f}^{(r)}=\left\{\Delta_{+}^{(r)} f(\alpha) \mid \alpha \in \mathbb{Z}_{2^{n}}\right\}, r \in\{1, \ldots, n-1\} .
$$

Отметим, что $\mathrm{B}_{r, 2^{n-r}}=\left\{f \in P\left(\mathbb{Z}_{2^{n}}\right) \mid d_{+}(f) \leq r\right\}$ состоит из всех отображений, $\Delta_{+}$-степень $d_{+}$которых не превосходит $r$. Кроме того, если $f \notin \mathrm{B}_{r-1,2^{n-r-1}}^{(n)}$ и $f \in \mathrm{B}_{r, 2^{n-r}}^{(n)}$, то $d_{+}(f)=r$.

Рассмотрим аффинную группу $A G L_{1}\left(\mathbb{Z}_{2^{n}}\right)$. Согласно утверждению 1 , для любой подстановки $g \in A G L_{1}\left(\mathbb{Z}_{2^{n}}\right)$ справедливо равенство $d_{+}(g)=n$. В частности, $\Delta_{+}$-степень $d_{+}$единичной подстановки $f(x)=x$ также равна $n$. Заметим, что таким же свойством обладает и единичная подстановка на $V_{n}$ относительно последовательности "классических" производных по любой последовательности различных переменных. 
Легко проверить, что очевидно сравнение

$$
\sum_{\beta \in C_{f}^{(r)}} \beta q \equiv 2_{2^{n}} \sum_{\alpha=0}^{2^{n-r}-1} f(\alpha) .
$$

Найдём число всех $(r, q)$-отображений, дадим их описание, а также укажем все пары $(r, q)$, для которых $\mathrm{B}_{r, q} \neq \varnothing$.

Утверждение 4. Пусть $q \in \mathbb{N}, r \in\{1, \ldots, n\}$. Тогда:

1) если $q \notin\left\{2^{t} \mid t \in Z_{n-r+1}\right\}$, mо $\mathrm{B}_{r, q}=\varnothing$;

2) если $q \in\left\{2^{t} \mid t \in Z_{n-r+1}\right\}$ ud $d=2^{n-r} / q$, mo

$$
\left|\mathrm{B}_{r, q}\right|=2^{n\left(2^{r}-1\right) 2^{n-r}}\left(\begin{array}{c}
2^{n} \\
d
\end{array}\right) 2^{n-r} !(q !)^{-d} .
$$

Доказательство. 1) Равенство $\mathrm{B}_{r, q}=\varnothing$ следует из соотношения $2^{n-r} \equiv_{q} 0$, так как согласно определению $\mathrm{B}_{r, q}, 2^{n} / 2^{r} q-$ число попарно различных значений, принимаемых $\Delta_{+}^{r} f$ для некоторого отображения $f \in P\left(\mathbb{Z}_{2^{n}}\right)$.

2) Рассмотрим произвольный упорядоченный набор $\beta=\left(\beta_{0}, \ldots, \beta_{d-1}\right) \in Z_{2^{n}}^{d}, \beta_{0}<\ldots<\beta_{d-1}$. Число всех таких наборов равно $w_{1}=\left(\begin{array}{c}2^{n} \\ d\end{array}\right)$. Для произвольного набора $\left(\delta_{0}, \ldots, \delta_{2^{n-r}-1}\right) \in Z_{2^{n}}^{2^{n-r}}$ число отображений $f: \mathbb{Z}_{2^{n}} \rightarrow \mathbb{Z}_{2^{n}}$, удовлетворяющих системе $\Delta_{+}^{r} f(i) \equiv_{2^{n}} \delta_{i}$, $i=0, \ldots, 2^{n-r}-1$, равно $w_{2}=2^{n\left(2^{r}-1\right) 2^{n-r}}$. Число наборов $\left(\delta_{0}, \ldots, \delta_{2^{n-r}-1}\right)$, для которых $\left\{\left\{\delta_{0}, \ldots, \delta_{2^{n-r}-1}\right\}\right\}$ - мультимножество первичной спецификации $\left[\beta_{0}^{q}, \ldots, \beta_{2^{n-r}-1}^{q}\right]$, равно $w_{3}=2^{n-r} ! /(q !)^{d}$. Таким образом,

$$
\left|\mathrm{B}_{r, q}\right|=w_{1} w_{2} w_{3}=2^{n\left(2^{r}-1\right) 2^{n-r}}\left(\begin{array}{c}
2^{n} \\
d
\end{array}\right) 2^{n-r} !(q !)^{-d} .
$$

Опишем свойства множества $\mathrm{B}_{r, q}$ для некоторых $r, q \in \mathbb{N}$.

Следствие 2. Пусть $q \in \mathbb{N}, r \in\{1, \ldots, n-1\}$. Тогда:

1) для любого отображения $g \in P\left(\mathbb{Z}_{2^{n}}\right)$ существуют такие $r^{\prime} \in$ $\{1, \ldots, n-1\}$ и $q^{\prime} \in \mathbb{N}$, что $g \in \mathrm{B}_{r^{\prime}, q^{\prime}}$, в частиости, $g \in B_{n-1,1} \cup$ $B_{n-1,2}$; 
2) $\mathrm{B}_{r, 2^{n-r}} \subset \mathrm{B}_{r+1,2^{n-r-1}}$;

3) $\mathrm{B}_{n-2,1} \cup \mathrm{B}_{n-2,2} \subset \mathrm{B}_{n-1,1} \cup \mathrm{B}_{n-1,2}$.

Доказательство. 1) Пусть $\Delta_{+}^{n-1} g(0) \equiv_{2^{n}} \beta_{0}, \quad \Delta_{+}^{n-1} g(1) \equiv_{2^{n}} \beta_{1}$. Если $\beta_{0} \equiv_{2^{n}} \beta_{1}$, то $g \in \mathrm{B}_{n-1,1}$, если же $\beta_{0} \equiv_{2^{n}} \beta_{1}$, то $g \in \mathrm{B}_{n-1,2}$.

2) Отметим, что

$$
\mathrm{B}_{j, 2^{n-j}}=\left\{f \in P\left(\mathbb{Z}_{2^{n}}\right) \mid \Delta_{+}^{(j)} f(0) \equiv{ }_{2^{n} \ldots} \equiv_{2^{n}} \Delta_{+}^{(j)} f\left(2^{n-j}-1\right)\right\}
$$

для каждого $j \in\{1, \ldots, n-1\}$. Если $g \in \mathrm{B}_{r, 2^{n-r}}$ и $\Delta_{+}^{(r)} g(0) \equiv_{2^{n}} \delta$ для некоторого $\delta \in \mathbb{Z}_{2^{n}}$, то

$$
\Delta_{+}^{(r+1)} g(\alpha) \equiv_{2^{n}} \Delta_{+}^{(r)} g(\alpha)+\Delta_{+}^{(r)} g\left(\alpha+2^{n-r-1}\right) \equiv_{2^{n}} 2 \delta
$$

для $\alpha=0, \ldots, 2^{n-r-1}-1$, т.е. $g \in \mathrm{B}_{r+1,2^{n-r-1}}$. Очевидно, что $\mathrm{B}_{r, 2^{n-r}} \neq$ $\mathrm{B}_{r+1,2^{n-r-1}}$. Значит, $\mathrm{B}_{r, 2^{n-r}} \subset \mathrm{B}_{r+1,2^{n-r-1}}$.

3) Если $g \in \mathrm{B}_{n-2,2^{c}}, c \in\{0,1,2\}$, и $\Delta_{+}^{(n-2)} g(i) \equiv{ }_{2^{n}} \delta_{i}, i=0, \ldots, 3$, то

$$
\Delta_{+}^{(n-1)} g(0) \equiv{ }_{2^{n}} \delta_{0}+\delta_{2}, \Delta_{+}^{(n-1)} g(1) \equiv{ }_{2^{n}} \delta_{1}+\delta_{3} .
$$

Возможны две ситуации: либо $\delta_{0}+\delta_{2} \not \equiv_{2^{n}} \delta_{1}+\delta_{3}$, либо $\delta_{0}+\delta_{2} \equiv_{2^{n}} \delta_{1}+\delta_{3}$. $\mathrm{B}$ первом случае имеет место включение $\mathrm{B}_{n-2,2^{c}} \subset \mathrm{B}_{n-1,1}$, а во втором случае включение $\mathrm{B}_{n-2,2^{c}} \subset \mathrm{B}_{n-1,2}$. Значит,

$$
\mathrm{B}_{n-2,1} \cup \mathrm{B}_{n-2,2} \subset \mathrm{B}_{n-1,1} \cup \mathrm{B}_{n-1,2} \text {. }
$$

Отображения из класса $\mathrm{B}_{r, 1}$ характеризуются тем, что число значений, принимаемых орбитальной производной $r$-го порядка, для каждого отображения этого класса является наибольшим (равным $2^{n-r}$ ) и каждое значение принимается наименьшее число $2^{r}$ раз. В этом смысле они являются аналогом отображений, разностная матрица которых имеет наибольшее число ненулевых элементов. В следующем утверждении доказывается существование таких отображений, что для всех $r \in\{1, \ldots, n-1\}$ орбитальная производная $r$-го порядка принимает наибольшее число значений. Также показывается, что в любом классе $\mathrm{B}_{r, 2}$ найдутся отображения, принадлежащие классу $\mathrm{B}_{r, 2^{c}}$ при $c \geq t$.

Утверждение 5. Пусть $q \in \mathbb{N}, r \in\{1, \ldots, n-1\}, n \geq 2$. Тогда:

$$
\text { 1) } \bigcap_{j=1}^{n-1} \mathrm{~B}_{j, 1} \neq \varnothing
$$


2) $\mathrm{B}_{r, 2^{t}} \cap \mathrm{B}_{r+1,2^{c}} \neq \varnothing$ npu $t \in\{0, \ldots, n-r\}, c \in\{t, \ldots, n-r-1\}$;

3) $\mathrm{B}_{r, 2^{t+1}} \cap \mathrm{B}_{r+1,2^{t}} \neq \varnothing$ npu $t \in\{0, \ldots, n-t-1\}$.

Доказательство. 1) Рассмотрим отображение $g \in P\left(\mathbb{Z}_{2^{n}}\right)$, удовлетворяющее равенствам $\Delta_{+}^{(1)} g(j-1)=j, j=1, \ldots, 2^{n-1}$. Отметим, что существование такого отображения следует из доказательства п.2 утверждения 4.

Индукцией по $r \in\{1, \ldots, n-1\}$ покажем справедливость соотношений

$$
\Delta_{+}^{(r)} g(j-1) \equiv{ }_{2^{n}} 2^{r-1} j+\sum_{j=2}^{r} 2^{n-j+(r-j)}, j=1, \ldots, 2^{n-r} .
$$

Если $r=2$, то

$$
\Delta_{+}^{(2)} g(j-1) \equiv{ }_{2^{n}} \Delta_{+}^{(1)} g(j-1)+\Delta_{+}^{(1)} g\left(j-1+2^{n-2}\right) \equiv{ }_{2^{n}} 2 j+2^{n-2}
$$

для $j=1, \ldots, 2^{n-2}$. Предположим, что

$$
\Delta_{+}^{(r)} g(j-1) \equiv{ }_{2^{n}} 2^{r-1} j+2^{n-2} \sum_{i=2}^{r} 2^{r-i}, j=1, \ldots, 2^{n-r} .
$$

Тогда

$$
\begin{aligned}
& \Delta_{+}^{(r+1)} g(j-1) \equiv{ }_{2^{n}} \Delta_{+}^{(r)} g(j-1)+\Delta_{+}^{(r)} g\left(j-1+2^{n-r-1}\right) \equiv_{2^{n}} \\
& \equiv_{2^{n}} 2^{r} j+2^{r-1+n-r-1}+\sum_{i=2}^{r} 2^{n-j+(r-i)+1} \equiv_{2^{n}} \\
& \equiv_{2^{n}} 2^{r} j+\sum_{i=2}^{r+1} 2^{n-j+(r-i)+1}, j=1, \ldots, 2^{n-r-1} .
\end{aligned}
$$

Так как

$$
2^{r-1} j_{1}+\sum_{i=2}^{r} 2^{n-i+(r-i)} \not \equiv 2^{n} 2^{r-1} j_{2}+\sum_{i=2}^{r} 2^{n-i+(r-i)}
$$

при любых различных $j_{1}, j_{2} \in\left\{1, \ldots, 2^{n-r}\right\}$, то $g \in \mathrm{B}_{r, 1}$ при $r=1, \ldots, n-1$, т.е. $\bigcap_{j=1}^{n-1} \mathrm{~B}_{j, 1} \neq \varnothing$.

2) Пусть $t \geq 1, \Delta_{+}^{(r)} g(j) \equiv 2_{2^{n}} \delta_{j}$ для $j=0, \ldots, 2^{n-r}-1$, и $\left\{\left\{\delta_{0}, \ldots, \delta_{2^{n-r}-1}\right\}\right\}$ - мультимножество с первичной спецификацией $\left[\lambda_{0}^{2^{t}}, \ldots, \lambda_{2^{n-r-t}-1}^{2^{t}}\right], \lambda_{i} \neq \lambda_{j}$ при $i \neq j$. Отметим, что $\Delta_{+}^{(r+1)} g(j) \equiv{ }_{2^{n}} \delta_{j}+$ $\delta_{j+2^{n-r-1}}$ для $j=0, \ldots, 2^{n-r-1}-1$. 
Пусть $d_{i, j}^{0}=\left\lfloor i / 2^{j}\right\rfloor, d_{i, s, j}^{1}=\left\lfloor\left(i-2^{s}\right) 2^{-j}\right\rfloor$ и

$$
\delta_{i} \equiv_{2^{n}} \begin{cases}\lambda_{d_{i, t-1}^{0}}, & \text { если } i<2^{n-r-1}, \\ \lambda_{2 d_{i, n-r-1, t}^{1}+1}, & \text { если } i \geq 2^{n-r-1}, d_{i, t-1}^{0} \equiv_{2} 0, \\ \lambda_{2 d_{i, n-r-1, t}^{1}}, & \text { если } i \geq 2^{n-r-1}, d_{i, t-1}^{0} \equiv_{2} 1 .\end{cases}
$$

Тогда $\Delta_{+}^{(r+1)} g(j) \equiv_{2^{n}} \lambda_{2 d_{j, t}^{0}}+\lambda_{2 d_{j, t}^{0}+1}$ для $j=0, \ldots, 2^{n-r-1}-1$. Положим

$$
\delta_{j}^{\prime} \equiv_{2^{n}} \Delta_{+}^{(r+1)} g(j), j=0, \ldots, 2^{n-r-1}-1 .
$$

Докажем существование таких элементов $\delta_{1}, \ldots, \delta_{2^{n-r}}$ (или, что равносильно, элементов $\left.\lambda_{0}, \lambda_{1}, \ldots, \lambda_{2^{n-r-t}-1}\right)$, что при некоторых попарно различных элементах $\beta_{0}, \ldots, \beta_{2^{n-r-c}-1}$ из кольца $\mathbb{Z}_{2^{n}}$ имеет место равенство $\beta_{l}=\delta_{2^{c} l+j}^{\prime}$ для $l=0, \ldots, 2^{n-r-c}-1, j=0, \ldots, 2^{c}-1$. Это означает, что $\mathrm{B}_{r, 2^{t}} \cap \mathrm{B}_{r+1,2^{c}} \neq \varnothing$.

Для $j \in\left\{0, \ldots, 2^{n-r-c}-1\right\}$ положим

$$
\left(\lambda_{2 i}, \lambda_{2 i+1}\right) \equiv_{2^{n}}\left(i+2^{c-t+1} j, 2^{c-t+1}(j+1)-i\right),
$$

где $i \in\left\{(j-1) \cdot 2^{c-t}, \ldots, j \cdot 2^{c-t}-1\right\}$. Нетрудно убедиться, что

$$
\lambda_{0}<\lambda_{1}<\ldots<\lambda_{2^{n-r-t}-1}, \lambda_{2 i}+\lambda_{2 i+1} \equiv{ }_{2^{n}} 2^{c-t+1} j+2^{c-t},
$$

где $i \in\left\{j 2^{c-t}, \ldots,(j+1) 2^{c-t}-1\right\}, j \in\left\{0, \ldots, 2^{n-r-c}-1\right\}$. Значит, $\beta_{j} \equiv 2_{2^{n}} 2^{c-t+1} j+2^{c-t}$ для $j \in\left\{0, \ldots, 2^{n-r-c}-1\right\}$, причём $\beta_{j_{1}} \not \equiv_{2^{n}} \beta_{j_{2}}$ при $j_{1} \neq j_{2}$ и $\mathrm{B}_{r, 2^{t}} \cap \mathrm{B}_{r+1,2^{c}} \neq \varnothing$ при $c \in\{t, \ldots, n-r-1\}$.

Для $t=0$ покажем, что существуют попарно различные элементы $\delta_{0}, \delta_{1}, \ldots, \delta_{2^{n-r}-1}$ со свойством

$$
\beta_{l} \equiv{ }_{2^{n}} \Delta_{+}^{(r+1)} g\left(2^{c} l+j\right) \equiv{ }_{2^{n}} \delta_{2^{c} l+j}+\delta_{2^{c} l+j+2^{n-r-1}},
$$

где $l=0, \ldots, 2^{n-r-c}-1, j=0, \ldots, 2^{c}-1$, для некоторых попарно различных элементов $\beta_{0}, \ldots, \beta_{2^{n-r-c-1}} \in \mathbb{Z}_{2^{n}}$.

Для некоторого $j \in\left\{0, \ldots, 2^{n-r-c}-1\right\} \quad$ и $\quad$ всех $i \in\left\{(j-1) \cdot 2^{c}, \ldots, j \cdot 2^{c}-1\right\}$ положим

$$
\left(\delta_{i}, \delta_{i+2^{n-r-1}}\right) \equiv_{2^{n}}\left(i+2^{c+1} j, 2^{c+1}(j+1)-i\right) .
$$

Нетрудно убедиться, что $\delta_{p_{1}} \neq \delta_{p_{2}}$ при $p_{1}, p_{2} \in\left\{0, \ldots, 2^{n-r}-1\right\}, p_{1} \neq p_{2}$, и что

$$
\delta_{2^{c} j+i}+\delta_{2^{c} j+i+2^{n-r-1}} \equiv_{2^{n}} 2^{c+1} j+2^{c},
$$


где $i \in\left\{j \cdot 2^{c}, \ldots,(j+1) \cdot 2^{c}-1\right\}, j \in\left\{0, \ldots, 2^{n-r-c}-1\right\}$. Таким образом, $\beta_{j} \equiv_{2^{n}} 2^{c+1} j+2^{c}$ для $j \in\left\{0, \ldots, 2^{n-r-c}-1\right\}$, причём $\beta_{j_{1}} \neq_{2^{n}} \beta_{j_{2}}$, если $j_{1} \neq j_{2}$. Значит, $\mathrm{B}_{r, 1} \cap \mathrm{B}_{r+1,2^{c}} \neq \varnothing$ при $c \in\{0, \ldots, n-r-1\}$.

3) Пусть $\Delta_{+}^{(r)} g(j) \equiv_{2^{n}} \delta_{j}, j=0, \ldots, 2^{n-r}-1$, и $\left\{\left\{\delta_{0}, \ldots, \delta_{2^{n-r}-1}\right\}\right\}$ - мультимножество с первичной спецификацией $\left[\lambda_{0}^{2^{t}}, \ldots, \lambda_{2^{n-r-t}-1}^{2^{t}}\right], 2 \lambda_{i} \neq_{2^{n}} 2 \lambda_{j}$ при $i \neq j$,

$$
\Delta_{+}^{(r+1)} g(j) \equiv_{2^{n}} \delta_{j}+\delta_{j+2^{n-r-1}}, j=0, \ldots, 2^{n-r-1}-1 .
$$

Для $i \in\left\{0, \ldots, 2^{n-r}-1\right\}$ положим

$$
\delta_{i} \equiv_{2^{n}} \begin{cases}\lambda_{d_{i, t-1}^{0}}, & \text { если } i<2^{n-r-1}, \\ \lambda_{d_{i, n-r-1, t-1}^{1}}, & \text { если } i \geq 2^{n-r-1} .\end{cases}
$$

Тогда $\Delta_{+}^{(r+1)} g(j) \equiv_{2^{n}} 2 \lambda_{j}$, причём $2 \lambda_{j}$ встречается ровно $2^{t-1}$ раз, где $j=$ $0, \ldots, 2^{n-r-1}-1$. Значит, $\mathrm{B}_{r, 2^{t+1}} \cap \mathrm{B}_{r+1,2^{t}} \neq \varnothing$.

Приведём примеры $r$-отображений для некоторых $r \in \mathbb{N}$.

Следствие 3. Пусть $f \in P\left(\mathbb{Z}_{2^{n}}\right)$.

1) $\operatorname{Ecлu~} f(x) \equiv_{2^{n}} a x+b,\|a\|=c \geq 0$, mo $f \in \mathrm{B}_{r, 2^{c+r}}$.

2) $\operatorname{Ecлu~} f(x) \equiv{ }_{2^{n}} \sum_{i=0}^{d} a_{i} x^{i}, a_{d} \neq 0 u$

$$
\sum_{i \in\{3, \ldots, d\}, i \equiv 1(\bmod 2)} a_{i} \equiv_{2} 0
$$

то $f$ есть $(n-2)$-отображение.

Доказательство. 1. Из леммы 5 следует, что

$$
\Delta_{+}^{(r)} f(x) \equiv{ }_{2^{n}} 2^{r} a x+2^{r} b+2^{n-1} a .
$$

Значит, $2^{r} a\left(x_{2}-x_{1}\right) \equiv{ }_{2^{n}} 0$, если

$$
2^{r} a x_{1}+2^{r} b+2^{n-1} a \equiv{ }_{2^{n}} 2^{r} a x_{2}+2^{r} b+2^{n-1} a .
$$

Очевидно, что сравнение $2^{r+c} a^{\prime} x \equiv{ }_{2^{n}} 0$ имеет $2^{r+c}$ решений, где $a=2^{c} a^{\prime}$, $a^{\prime}$ нечётно. Таким образом, $f \in \mathrm{B}_{r, 2^{c+r}}$.

2. Из утверждения 1 имеем

$$
\Delta_{+}^{(n-2)} f(x) \equiv{ }_{2^{n}} 2^{n-2} f(x)+2^{n-1} f^{\prime}(x),
$$


где $f^{\prime}(x) \equiv_{2^{n}} \sum_{i=0}^{d-1}(i+1) a_{i+1} x^{i}-$ "классическая" производная, и отображение $\Delta_{+}^{(n-2)} f(x)$ принимает значения из множества $\left\{0,2^{n-2}, 2^{n-1}, 3 \cdot 2^{n-2}\right\}$. Отметим, что число решений уравнения $\Delta_{+}^{(n-2)} f(x) \equiv{ }_{2^{n}} 2^{n-2} \beta$ для $\beta \in$ $Z_{4}$ равно

$$
2^{n-2} \cdot\left|\left\{\gamma \in Z_{4} \mid f(\gamma)+2 f^{\prime}(\gamma) \equiv_{4} \beta\right\}\right|
$$

Рассмотрим отображение $g: \mathbb{Z}_{4} \rightarrow \mathbb{Z}_{4}$, где

$$
g(x) \equiv_{4} f(x)+2 f^{\prime}(x) .
$$

Нетрудно убедиться в том, что

$$
\begin{aligned}
& \quad g(0) \equiv_{4} a_{0}+2 a_{1}, \quad g(2) \equiv_{4} a_{0}+2 a_{1}+2 a_{1} \equiv_{4} a_{0}, \\
& g(1) \equiv_{4} \sum_{i=0}^{d-1} a_{i}+\sum_{i=0}^{d-1} 2 i a_{i} \equiv_{4} \\
& \equiv_{4} \sum_{i \in\{0, \ldots, d-1\}, i \equiv 0(\bmod 2)} a_{i}+\sum_{i \in\{0, \ldots, d-1\}, i \equiv 1(\bmod 2)} a_{i}(2 i+1), \\
& g(3) \equiv_{4} \sum_{i=0}^{n-1} a_{i}(-1)^{i}+\sum_{i=0}^{n-1} 2 i a_{i}(-1)^{i-1} \equiv_{4} \\
& \equiv_{4} \sum_{i \in\{0, \ldots, d-1\}, i \equiv 0(\bmod 2)} a_{i}+\sum_{i \in\{0, \ldots, d-1\}, i \equiv 1(\bmod 2)} a_{i}(2 i-1),
\end{aligned}
$$

причём

$$
g(1)-g(3) \equiv_{4} 2 \sum_{i \in\{0, \ldots, d-1\}, i \equiv 1(\bmod 2)} a_{i}, \quad g(2)-g(0) \equiv_{4} 2 a_{1} .
$$

Пусть

$$
\tilde{a} \equiv_{4} \sum_{i \in\{0, \ldots, d-1\}, i \equiv 1(\bmod 2)} a_{i} .
$$

Из соотношений (12) следует, что если числа $\tilde{a}$ и $a_{1}$ оба чётны, то $g(1) \equiv_{4}$ $g(3)$ и $g(2) \equiv_{4} g(0)$, если же оба числа нечётны, то $g(1) \not_{4} g(3)$ и $g(2) \not_{4} g(0)$. Кроме того, $g(1) \equiv_{2} g(3)$ и $g(2) \equiv_{2} g(0)$. Следовательно, $\Delta_{+}^{n-2} g(x)$ принимает на $Z_{4}$ каждое значение одинаковое число раз, т.е. $f$ является $(n-2)$-отображением.

В доказательстве утверждения 4 найдена мощность множества $\mathrm{B}_{r, 2^{n-r}}^{(n)}$ и приведены все преобразования из этого множества. Задача нахождения всех биекций множества $\mathrm{B}_{r, 2^{n-r}}^{(n)}$ значительно сложнее. 
Положим $\mathrm{B}_{r, 2^{n-r}}^{(s)}=\mathrm{B}_{r, 2^{n-r}} \cap S\left(\mathbb{Z}_{2^{n}}\right), r \in\{1, \ldots, n-1\}$. Опишем множество $\mathrm{B}_{1,2^{n-1}}^{(s)}$.

Для $\gamma \in Z_{2^{n}}, f \in P\left(\mathbb{Z}_{2^{n}}\right)$ положим

$$
\begin{gathered}
U^{(1)}(f)=\left\{\left\{f(\alpha), f\left(\alpha+2^{n-1}\right)\right\} \mid \alpha \in Z_{2^{n-1}}\right\}, \\
\Gamma_{\gamma}^{(1)}={ }_{2^{n}}\left\{\{\beta, \gamma-\beta\} \mid \beta \in \mathbb{Z}_{2^{n}}\right\} .
\end{gathered}
$$

Очевидно, что тогда и только тогда $f \in \mathrm{B}_{1,2^{n-1}}^{(s)}$, когда $U^{(1)}(f)=\Gamma_{\gamma}$ для некоторого нечётного $\gamma \in Z_{2^{n}}$. Кроме того, $\mathrm{B}_{1,2^{n-1}}^{(s)} \subset \mathrm{B}_{2,2^{n-2}}^{(s)}$.

Для $\gamma \in Z_{2^{n-1}}, \delta \in Z_{2^{n}}, f \in P\left(\mathbb{Z}_{2^{n}}\right)$ положим

$$
\begin{aligned}
& U^{(2)}(f)=\left\{\left\{f\left(\alpha+2^{n-2} c\right) \mid c \in Z_{4}\right\} \mid \alpha \in Z_{2^{n-2}}\right\}, \\
& \delta^{[\gamma]} \equiv_{2^{n}} \begin{cases}\gamma-\delta, & \text { если } \delta \in\{0, \ldots, \gamma\}, \\
\gamma-\delta+2^{n-1}, & \text { если } \delta \in\left\{\gamma+1, \ldots, 2^{n}-1\right\} .\end{cases} \\
& \lambda_{\gamma}(\delta)= \begin{cases}0, & \text { если } \gamma \geq \delta, \\
1, & \text { если } \gamma<\delta .\end{cases}
\end{aligned}
$$

Для $\gamma \in Z_{2^{n-1}}$ рассмотрим множество

$$
\begin{aligned}
& \Gamma_{\gamma}^{(2)}=2^{n} \\
& =2^{n}\left\{\left\{\beta, \beta^{[\gamma]}, \beta^{\prime}+2^{n-1}, \beta^{\prime[\gamma]}+2^{n-1}\right\} \mid \beta, \beta^{\prime} \in Z_{2^{n-1}}, \lambda_{\gamma}(\beta)=\lambda_{\gamma}\left(\beta^{\prime}\right)\right\} .
\end{aligned}
$$

Утверждение 6. 1) Пусть нечётный элемент $\gamma \in Z_{2^{n-1}}$ и подстановка $f \in S\left(\mathbb{Z}_{2^{n}}\right)$ удовлетворлют равенству $U^{(2)}(f)=\Gamma_{\gamma}^{(2)}$. Тогда $f \in \mathrm{B}_{2,2^{n-2}}^{(s)}$. Кроме того, $f \notin \mathrm{B}_{1,2^{n-1}}^{(s)}$.

2) Число подстановок, удовлетворяющих условиям п.1, равно

$$
\sum_{\gamma=0}^{2^{n-2}-1}(2 \gamma+1) !\left(2^{n-1}-2 \gamma+1\right) !
$$

Доказательство. 1. Условие $\gamma \equiv_{4} 2$ получается из леммы 2. Очевидно, что $\beta^{[\gamma]} \in Z_{2^{n-1}}$ и

$$
\beta^{[\gamma]}+\beta \equiv_{2^{n}} \begin{cases}\gamma, & \text { если } \gamma \geq \beta, \\ \gamma+2^{n-1}, & \text { если } \gamma<\beta,\end{cases}
$$


a $\left\{\left\{\beta, \beta^{[\gamma]}\right\} \mid \beta \in Z_{2^{n-1}}\right\}-$ разбиение множества $Z_{2^{n-1}}$.

Аналогично для элемента $\beta \in Z_{2^{n-1}}$ имеем

$$
M_{\beta}=\left\{\beta+2^{n-1}, \beta^{[\gamma]}+2^{n-1}\right\}_{2^{n}} \subset\left\{2^{n-1}, \ldots, 2^{n}-1\right\},
$$

и $\left\{M_{\beta} \mid \beta \in Z_{2^{n-1}}\right\}$ есть разбиение множества $\left\{2^{n-1}, \ldots, 2^{n}-1\right\}$, а $\Gamma_{\gamma}^{(2)}$ разбиение $Z_{2^{n}}$. Аналогично

$$
\beta^{[\gamma]}+2^{n-1}+\beta+2^{n-1} \equiv_{2^{n}} \begin{cases}\gamma, & \text { если } \gamma \geq \beta, \\ \gamma+2^{n-1}, & \text { если } \gamma<\beta,\end{cases}
$$

Таким образом, если $\beta, \beta^{\prime} \in Z_{2^{n-1}}$, то

$$
\begin{gathered}
\beta+\beta^{[\gamma]}+\beta^{\prime}+2^{n-1}+\beta^{\prime[\gamma]}+2^{n-1} \equiv_{2^{n}} \\
\equiv_{2^{n}} \begin{cases}2 \gamma, & \text { если } \lambda_{\gamma}(\beta)=\lambda_{\gamma}\left(\beta^{\prime}\right), \\
2 \gamma+2^{n-1}, & \text { если } \lambda_{\gamma}(\beta) \neq \lambda_{\gamma}\left(\beta^{\prime}\right) .\end{cases}
\end{gathered}
$$

Кроме того, если $U^{(2)}(f)=\Gamma_{\gamma}^{(2)}$, то для любого $\alpha \in Z_{2^{n-2}}$ имеем

$$
\sum_{c \in Z_{4}} f\left(\alpha+2^{n-2} c\right) \equiv 2^{n} 2 \gamma
$$

Так как $\Gamma_{\gamma}^{(2)}-$ разбиение $Z_{2^{n}}$, то $f \in \mathrm{B}_{2,2^{n-2}}^{(s, n)}$.

Покажем, что $f \notin \mathrm{B}_{1,2^{n-1}}^{(s, n)}$. Предположим, что $f \in \mathrm{B}_{1,2^{n-1}}^{(s, n)}$. Тогда из сравнения (13) для некоторых $\delta, \delta^{\prime} \in Z_{2^{n}}$ и $\beta, \beta^{\prime} \in Z_{2^{n-1}}, \lambda_{\gamma}(\beta)=\lambda_{\gamma}\left(\beta^{\prime}\right)$, имеем

$$
\left\{\beta, \beta^{[\gamma]}, \beta^{\prime}+2^{n-1}, \beta^{\prime[\gamma]}+2^{n-1}\right\}={ }_{2^{n}} B
$$

где $B=2_{2^{n}}\left\{\delta, 2 \gamma-\delta, \delta^{\prime}, 2 \gamma-\delta^{\prime}\right\}$.

Так как

$$
\beta+\beta^{[\gamma]} \in{2^{n}}\left\{\gamma, \gamma+2^{n-1}\right\}, \beta^{\prime}+\beta^{\prime[\gamma]} \in_{2^{n}}\left\{\gamma, \gamma+2^{n-1}\right\},
$$

то $\beta+\beta^{\prime}+2^{n-1} \equiv_{2^{n}} 2 \gamma$ или $\beta+\beta^{\prime[\gamma]}+2^{n-1} \equiv_{2^{n}} 2 \gamma$.

Если $\beta+\beta^{\prime}+2^{n-1} \equiv_{2^{n}} 2 \gamma$, то $\beta^{[\gamma]}+\beta^{\prime[\gamma]}+2^{n-1} \equiv_{2^{n}} 2 \gamma$, но

$$
\beta^{[\gamma]}+\beta^{\prime[\gamma]}+2^{n-1} \equiv_{2^{n}} 2 \gamma-\beta-\beta^{\prime}+2^{n-1} \equiv_{2^{n}} 2^{n-1} .
$$

Если $\beta+\beta^{\prime[\gamma]}+2^{n-1} \equiv_{2^{n}} 2 \gamma$, то $\beta^{[\gamma]}+\beta+2^{n-1} \equiv_{2^{n}} 2 \gamma$, но

$$
\beta+\beta^{\prime[\gamma]}+2^{n-1} \equiv_{2^{n}} \begin{cases}\beta+\gamma-\beta^{\prime}+2^{n-1}, & \text { если } \gamma \geq \beta^{\prime}, \\ \gamma-\beta^{\prime}+\beta, & \text { если } \gamma<\beta^{\prime},\end{cases}
$$




$$
\beta^{[\gamma]}+\beta^{\prime}+2^{n-1} \equiv_{2^{n}} \begin{cases}\gamma-\beta+\beta^{\prime}+2^{n-1}, & \text { если } \gamma \geq \beta, \\ \gamma-\beta+\beta^{\prime}, & \text { если } \gamma<\beta .\end{cases}
$$

Так как $\lambda_{\gamma}(\beta)=\lambda_{\gamma}\left(\beta^{\prime}\right)$, то из сравнений $(14),(15)$ получаем, что при $\gamma \geq \beta^{\prime} \geq 0$ (тогда $\gamma \geq \beta$ ) должны выполняться сравнения

$$
\beta+\gamma-\beta^{\prime}+2^{n-1} \equiv 2_{2^{n}} 2 \gamma, \gamma-\beta+\beta^{\prime}+2^{n-1} \equiv_{2^{n}} 2 \gamma
$$

При $\gamma=0$ и $\beta^{\prime}-\beta \equiv 2_{2^{n}} 2^{n-1}$ или $\gamma=2^{n-1}, \beta=\beta^{\prime}$ возникает противоречие. Если $\gamma<\beta^{\prime}$ (тогда $\gamma<\beta$ ), то должны выполняться сравнения

$$
\gamma-\beta^{\prime}+\beta \equiv_{2^{n}} 2 \gamma, \gamma-\beta+\beta^{\prime} \equiv_{2^{n}} 2 \gamma,
$$

При $\gamma=0$ и $\beta^{\prime}=\beta$ или $\gamma=2^{n-1}, \beta \equiv_{2^{n}} \beta^{\prime}+2^{n-1}$ возникает противоречие. Значит, $f \notin \mathrm{B}_{1,2^{n-1}}^{(s)}$.

Доказательство п.2 следует из доказательства п.1 утверждения 4.

Для элементов $\alpha, x \in Z_{2^{n}}$ положим

$$
\delta_{\alpha}(x)= \begin{cases}1, & \text { если } \alpha=x, \\ 0, & \text { если } \alpha \neq x .\end{cases}
$$

Пусть

$$
\mathrm{B}_{r}^{(n)}=\left\{g \in P\left(\mathbb{Z}_{2^{n}}\right) \mid d_{+}(g) \leq r\right\}, r \in\{1, \ldots, n\} .
$$

Тогда из доказательства п. 2 утверждения 4 следует, что любое отображение $g_{u} \in \mathrm{B}_{r}^{(n)}$, т.е. отображение со свойством $\Delta_{+}^{(r)} g_{u}(x) \equiv_{2^{n}} 2^{r} u$, где $u \in Z_{2^{n}}, r \in\{1, \ldots, n\}$, однозначно представимо в виде

$$
\begin{gathered}
g_{u}(x) \equiv_{2^{n}} \\
\equiv_{2^{n}} \sum_{\beta \in Z_{2^{n-r}}}\left(\left(u-\sum_{\lambda \in U_{n, n-t}(\beta) \backslash\{\beta\}} c_{\lambda}\right) \delta_{\beta}(x)+\sum_{\lambda \in U_{n, n-t}(\beta) \backslash\{\beta\}} c_{\lambda} \delta_{\lambda}(x)\right)
\end{gathered}
$$

для некоторых $c_{2^{n-r}}, \ldots, c_{2^{n}-1} \in \mathbb{Z}_{2^{n}}$.

Пусть $g, g^{\prime} \in \mathrm{B}_{r}^{(n)}$ и $\Delta_{+}^{(r)} g(x) \equiv_{2^{n}} 2^{r} u, \Delta_{+}^{(r)} g^{\prime}(x) \equiv_{2^{n}} 2^{r} u^{\prime}$ для некоторых $u, u^{\prime} \in Z_{2^{n}}$. Очевидно, что $2 \leq \chi\left(g, g^{\prime}\right) \leq 2^{n}$. Кроме того, для произвольных элементов $c, c^{\prime} \in Z_{2^{n}}$ и отображения

$$
g_{c, c^{\prime}}: x \mapsto c \cdot g(x)+c^{\prime} \cdot g^{\prime}(x)\left(\bmod 2^{n}\right)
$$


из $P\left(\mathbb{Z}_{2^{n}}\right)$ выполняется сравнение $\Delta_{+}^{(r)} g_{c, c^{\prime}}(x) \equiv{ }_{2^{n}} 2^{r}\left(c u+c^{\prime} u^{\prime}\right)$. Значит, $g_{c, c^{\prime}}(x) \in \mathrm{B}_{r}^{(n)}$ и $\left(\mathrm{B}_{r}^{(n)},+\right)$ - абелева группа.

Таким образом, $\mathrm{B}_{r}^{(n)}$ является модулем над $Z_{2^{n}}$. Поэтому $\mathrm{B}_{r}^{(n)}$ можно рассматривать как аналог множества всех двоичных функций на $V_{n}$, степень которых не превосходит $r$.

Особо отметим свойства преобразований из множеств $\mathrm{B}_{r, 2^{n-r}}$ и $\mathrm{B}_{r, 1}$. Так, принадлежность к множеству $\mathrm{B}_{r, 1}$ означает, что число различных значений, принимаемых $r$ - й орбитальной производной, равно $2^{n-r}$ и является наибольшим. Принадлежность же к множеству $\mathrm{B}_{r, 2^{n-r}}$ означает, что $r$-я орбитальная производная принимает только одно значение.

Опишем подстановки из множества $\mathrm{B}_{1,1}$. Для элементов $\beta_{0}, \ldots, \beta_{2^{n-1}-1}$, удовлетворяющих равенству

$$
\left\{\beta_{0}, \ldots, \beta_{2^{n-1}-1}\right\}=\left\{2 i \mid i \in Z_{2^{n-1}}\right\},
$$

укажем такие разбиения $\mathrm{A}=\left(A_{0}, \ldots, A_{2^{n}-1}\right)$ множества $Z_{2^{n}}$ с блоками мощности 2 , что $\beta(\mathrm{A})=\left(\beta_{0}, \ldots, \beta_{2^{n-1}-1}\right)$. Способ построения данных разбиений основан на использовании линейного конгруэнтного генератора.

Утверждение 7. Пусть $\delta_{0} \in Z_{2^{n}}, a, b \in Z_{2^{n}},\|a\|=\|b\|=0,\|a-1\| \geq 2$, $f \in S\left(\mathbb{Z}_{2^{n}}\right), f(x) \equiv_{2^{n}}$ ax $+b u g \in S\left(\mathbb{Z}_{2^{n}}\right)$ обладает свойством

$$
\left\{g(\alpha), g\left(\alpha+2^{n-1}\right) \mid \alpha \in Z_{2^{n-1}}\right\}=\left\{\left\{\delta_{j}, \delta_{j+2^{n-1}}\right\} \mid j \in Z_{2^{n-1}}\right\} .
$$

Тогда $g \in \mathrm{B}_{1,1}$, где $\delta_{j}=f\left(\delta_{j-1}\right), j=1, \ldots, 2^{n}$.

Доказательство. Если $\|a\|=\|b\|=0$, то согласно [16] период линейного конгруэнтного генератора $f: \delta_{j-1} \mapsto \delta_{j}, j=0,1, \ldots$, равен $2^{n}$, в частности, $\left\{\delta_{i} \mid i \in Z_{2^{n}}\right\}=Z_{2^{n}}$. Кроме того,

$$
\delta_{t+j} \equiv_{2^{n}} a^{j} \delta_{t}+b\left(a^{j-1}+\ldots+a+1\right), t \in Z_{2^{n-1}}, j \in\left\{1, \ldots, 2^{n-1}\right\} .
$$

Значит,

$$
\delta_{t}+\delta_{t+2^{n-1}} \equiv_{2^{n}}\left(a^{2^{n-1}}+1\right) \delta_{t}+b\left(a^{2^{n-1}-1}+\ldots+a+1\right) .
$$

Предположим, что для некоторых элементов $t^{\prime}, t \in Z_{2^{n-1}}, t^{\prime} \geq t$, имеем

$$
\delta_{t}+\delta_{t+2^{n-1}} \equiv_{2^{n}} \delta_{t^{\prime}}+\delta_{t^{\prime}+2^{n-1}} .
$$

Так как $c^{2^{n-1}}+1 \equiv_{4} 2$ для любого нечётного $c \in \mathbb{N}$, то сравнение

$$
\left(a^{2^{n-1}}+1\right)\left(\delta_{t^{\prime}}-\delta_{t}\right) \equiv_{2^{n}} 0
$$


выполняется тогда и только тогда, когда $\delta_{t^{\prime}}-\delta_{t} \equiv_{2^{n-1}} 0$. Значит,

$$
\left(a^{j}-1\right) \delta_{t}^{\prime \prime}+b\left(a^{j-1}+\ldots+a+1\right) \equiv_{2^{n-1}} 0, j=t^{\prime}-t,
$$

причём $j<2^{n-1}$. Тогда сравнение (17) переписывается в виде

$$
(a-1) \cdot\left(a^{j-1}+\ldots+a+1\right) \delta_{t}+b \cdot\left(a^{j-1}+\ldots+a+1\right) \equiv_{2^{n-1}} 0,
$$

т.e.

$$
\left(a^{j-1}+\ldots+a+1\right)\left((a-1) \delta_{t}+b\right) \equiv_{2^{n-1}} 0 .
$$

Так как $\|a\|=\|b\|=0$, то $\left\|(a-1) \delta_{t}+b\right\|=0$ для любого $\delta_{t}$. Значит,

$$
a^{j-1}+\ldots+a+1 \equiv_{2^{n-1}} 0 .
$$

Заметим, что если $q, m \in \mathbb{N}$ и $a^{q-1}+\ldots+a+1 \equiv_{2^{m}} 0$, то $q=2 q_{1}$ и $a^{q-1}+$ $\ldots+a+1=\left(a^{q_{1}-1}+\ldots+a+1\right)\left(a^{q_{1}}+1\right) \equiv_{2^{m-1}} 0$.

Так как $\|a-1\| \geq 2$, то $\left\|a^{q_{1}}-1\right\| \geq 2$. Поэтому $\left\|a^{q_{1}-1}+\ldots+a+1\right\| \geq$ $m-1$. Таким образом, сравнение $(18)$ имеет место тогда и только тогда, когда $j=2^{n-1}$, но $j<2^{n-1}$. Значит,

$$
\left|\left\{\left(a^{2^{n-1}}+1\right) \delta_{t}+b\left(a^{2^{n-1}-1}+\ldots+a+1\right) \mid t \in Z_{2^{n-1}}\right\}\right|={ }_{2^{n}} 2^{n-1} .
$$

Положим $A_{j}=\left\{\delta_{j}, \delta_{j+2^{n-1}}\right\}, j=0, \ldots, 2^{n-1}-1$. Тогда разбиению $\mathrm{A}=\left(A_{0}, \ldots, A_{2^{n}-1}\right)$ соответствуют подстановки из $\mathrm{B}_{1,1}$, удовлетворяющие сравнению (16).

Из утверждения 17 следует, что для подстановки $g \in \mathrm{B}_{1,1}$, удовлетворяющей соотношению (16), множеством значений орбитальной производной первого порядка является

$$
\begin{aligned}
& \left\{\Delta_{+}^{(1)} g(\alpha) \mid \alpha \in Z_{2^{n-1}}\right\}={ }_{2^{n}} \\
& 2^{n}=\left\{\left(a^{2^{n-1}}+{ }_{2^{n}} 1\right) \delta_{t}+{ }_{2^{n}} b\left(a^{2^{n-1}-1}+{ }_{2^{n}} \ldots+{ }_{2^{n}} a+{ }_{2^{n}} 1\right) \mid t \in Z_{2^{n-1}}\right\} .
\end{aligned}
$$

В частности, при $a=1$ имеем

$$
\left\{\Delta_{+}^{(1)} g(\alpha) \mid \alpha \in Z_{2^{n-1}}\right\}=\left\{2 i \mid i \in Z_{2^{n-1}}\right\} .
$$

Приведём ещё один пример подстановок из множества $\mathrm{B}_{r, 1}$. Для $r \in\{1, \ldots, n\}$ положим

$C^{(r)}=\left\{g \in S(X) \mid\left\{g(\beta) \mid \beta \in U_{n, n-r}(\alpha)\right\}=U_{n, n-r}(\alpha), \alpha=0, \ldots, 2^{n-r}-1\right\}$. 
Так как для любой подстановки $g \in C^{(r)}$ имеем

$$
\Delta_{+}^{(r)} g(\alpha) \equiv_{2^{n}} \sum_{\beta \in U_{n, n-r}(\alpha)} g(\beta) \equiv_{2^{n}} \sum_{\beta \in U_{n, n-r}(\alpha)} \beta \equiv_{2^{n}} 2^{r} \alpha+2^{n-1},
$$

то $C^{(r)} \subseteq \mathrm{B}_{r, 1}$. Множество $C^{(r)}$ содержит единичную подстановку, циклы длины $2^{j}$, состоящие из всех элементов множества $U_{n, n-j}(\alpha), j \leq r, \alpha \in$ $Z_{2^{n-r}}$, и их произведений.

Отметим, что для любых чисел $r \in\{1, \ldots, n\}$, нечётного $b \in Z_{2^{n}}$ множество $\mathrm{B}_{r, 1}$ содержит полноцикловую подстановку $s^{(b)} \in S_{2^{n}}$, причём $\Delta_{+}^{(r)} s^{(b)}(x) \equiv{ }_{2^{n}} 2^{r}(x+b)$.

\section{Список литературы}

[1] Lai X., "Higher order derivatives and differential cryptanalysis.", Communications and Cryptography, 1994, 227-233.

[2] Aumasson J.-P., Kaasper E., Knudsen L. R., Matusiewicz K., Odegard R., Peyrin T., Schlaffer M., "Distinguishers for the compression function and output transformation of Hamsi-256", Lect. Notes Comput. Sci, 6168 (2010), 87-103.

[3] Boura C., Canteaut A., De Canniere C., "Higher-order differential properties of Keccak and Luffa", Lect. Notes Comput. Sci., 6733 (2011), 252-269.

[4] Knudsen L. R., "Truncated and higher order differentials.", Lect. Notes Comput. Sci., 1008 (1995), 196-211.

[5] Moriai S., Shimoyama T., Kaneko T., "Higher order differential attack of a CAST cipher", Lect. Notes Comput. Sci., 1372 (1998), 17-31.

[6] Canteaut A., Videnau M., "Degree of composition of highly nonlinear functions and applications to higher order differential cryptanalysis", Lect. Notes Comput. Sci., 2332 (2002), 518-533.

[7] Марков Д. А., Исчисление конечных разностей, 2 изд., Одесса, 1910.

[8] Гельфонд А. О., Исчисление конечных разностей, Москва, 1967.

[9] Березин И. С, Жидков Н. П., Методы вычислений, Т. 1, 2, 3 изд., Москва, 1966.

[10] Сачков В. Н., Введение в комбинаторные методы дискретной математики, М.: МЦНМО, 2004.

[11] Черемушкин А. В., “Аддитивный подход к определению степени нелинейности дискретной функции", Прикладная дискретная математика, 8:2 (2010), 22-33.

[12] Дискретная математика. Энииклопедия, М.: Научное издательство "Большая Российская энциклопедия", 2004.

[13] Iwata T., Kurosawa K., "Probabilistic higher order differential attack and higher order bent functions", Lect. Notes Comput. Sci., 1716 (1999), 62-74.

[14] Wagner D., "A generalized birthday problem", Lect. Notes Comput. Sci., 2442 (2002), 288304.

[15] Глухов М.М., Круглов И.А., Пичкур А.Б., Черемушкин А.В., Введение в теоретикочисловые методы в криптографии, С-Пб.: Лань, 2011.

[16] Кнут Д., Искусство программирования. Т. 2. Получисленные методы, М.: ООО "И.Д. Вильямс", 2007. 